\title{
The homeobox transcription factor VentX controls human macrophage terminal differentiation and proinflammatory activation
}

\author{
Xiaoming Wu, ${ }^{1}$ Hong Gao, ${ }^{1}$ Weixiong Ke, ${ }^{1}$ Roger W. Giese, ${ }^{2}$ and Zhenglun Zhu ${ }^{1}$ \\ 'Department of Medicine, Gastroenterology Division, Brigham and Women's Hospital, Harvard Medical School, Boston, Massachusetts, USA. \\ 2Department of Pharmaceutical Sciences, Northeastern University, Boston, Massachusetts, USA.
}

\begin{abstract}
Macrophages are critical players in both innate and adaptive immunity. While the exogenous signaling events leading to the terminal differentiation of macrophages from monocytes have been studied extensively, the underlying intracellular transcriptional mechanisms remain poorly understood. Here we report that the homeobox transcription factor Vent $X$ plays a pivotal role in human macrophage terminal differentiation and proinflammatory function. Our study showed that VentX expression was upregulated upon human primary monocyte-to-macrophage differentiation induced by cytokines such as M-CSF, GM-CSF, and IL-3. Moreover, ablation of VentX expression in primary monocytes profoundly impaired their differentiation to macrophages, and ectopic expression of Vent $X$ in a myeloid progenitor cell line triggered its differentiation with prominent macrophage features. Further analysis revealed that VentX was pivotal for the proinflammatory response of terminally differentiated macrophages. Mechanistically, VentX was found to control expression of proteins key to macrophage differentiation and activation, including M-CSF receptor. Importantly, preliminary analysis of gene expression in leukocytes from patients with autoimmune diseases revealed a strong correlation between levels of Vent $X$ and those of proinflammatory cytokines. Our results provide mechanistic insight into the crucial roles of Vent $X$ in macrophage differentiation and proinflammatory activation and suggest that dysregulation of Vent $X$ may play a role in the pathogenesis of autoimmune diseases.
\end{abstract}

\section{Introduction}

Macrophages play critical roles in both innate and adaptive immunity in virtually all tissues (1-3). Tissue macrophages are derived from circulating monocytes in response to microenvironmental factors such as M-CSF, GM-CSF, and IL-3 during extravascularization $(1,4)$. The process of monocyte-to-macrophage terminal differentiation remains a subject of extensive investigation in the contexts of immune defense against pathogen invasion, pathogenesis of autoimmune and inflammatory diseases, and carcinogenesis of hematopoietic and other malignancies $(1,4,5)$. Upon differentiation, macrophages can be further activated by extracellular signals and display diverse functional patterns depending upon the cytokines and microbial products present in the microenvironment. Macrophage activation has been classified into a classical pathway and an alternative pathway. In response to Th1 cytokines, such as IFN- $\gamma$ and LPS, macrophages display a classical activation phenotype and produce mainly proinflammatory cytokines. The Jak/Stat and activator protein 1 (AP-1)/NF- $\mathrm{KB}$ signaling pathways have been shown to play critical roles in classical activation of macrophages. Alternatively, macrophages can be activated by Th2 cytokines, such as IL-4 and IL-13, and exhibit distinct functions with antiinflammatory and tissue repair properties $(2,3,6,7)$.

The common myeloid progenitor cells are the bone marrow precursors of monocytes and macrophages. It is generally accepted that monocyte and macrophage development occurs by changes of transcriptional programs in a stepwise manner (8-11). Genetic studies with knockout mice have revealed the important roles of transcription factors such as PU.1 and CCAAT enhancer-binding protein $\alpha$ $(\mathrm{C} / \mathrm{EBP} \alpha)$ in monocyte/macrophage lineage commitment $(1,5,8$,

Conflict of interest: The authors have declared that no conflict of interest exists. Citation for this article: J Clin Invest. 2011;121(7):2599-2613. doi:10.1172/JCI45556.
12-14). Recently, global transcriptome analysis revealed profound changes in gene expression during monocyte-to-macrophage terminal differentiation $(15,16)$. Previous studies on human monocyte-to-macrophage differentiation have mainly relied on myeloid progenitor cell lines such as U937 and THP-1 (17-19). The key transcriptional mechanism controlling primary human monocyte-tomacrophage differentiation remains poorly defined.

Developmental modeling is informative in defining genes and pathways involved in host defense and immune regulation. Using methods of reverse genetics, we recently demonstrated that VentX, a human homolog of the Xenopus homeobox transcriptional factor Xom, is a LEF/TCF-associated Wnt repressor and a putative tumor suppressor $(20,21)$. We have also shown that VentX transactivates $\mathrm{p} 53 / \mathrm{p} 21$ and $\mathrm{p} 16^{\mathrm{ink} 4 a} / \mathrm{Rb}$ pathways to regulate senescence in tumor cells (22). VentX is predominantly expressed in hematopoietic cells and highly conserved in primates. However, we and others failed to identify the murine homolog of VentX in the current mouse genome database $(20,23,24)$.

In this study, we report that VentX plays an essential role in human primary monocyte-to-macrophage terminal differentiation and is required for optimal proinflammatory response during classical macrophage activation. Clinical data show that the expression level of VentX correlates positively with the expression levels of several proinflammatory cytokines, suggesting a potential role for VentX in the pathogenesis of inflammatory diseases.

\section{Results}

VentX expression is upregulated during monocyte-to-macrophage differentiation. Tissue expression profiling showed that VentX is expressed in monocytes (20). To explore the potential role of VentX in monocyte-to-macrophage differentiation, we examined VentX 


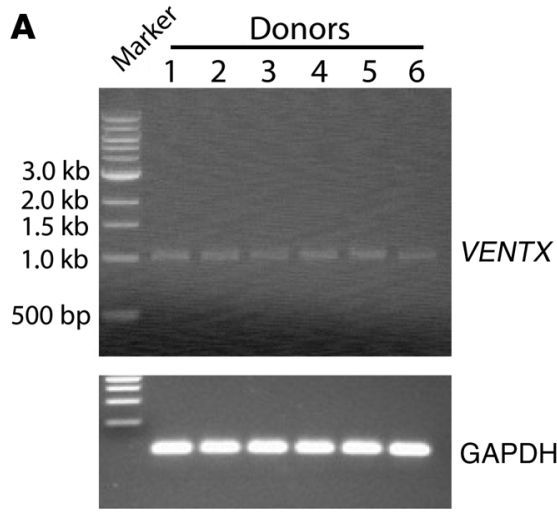

C

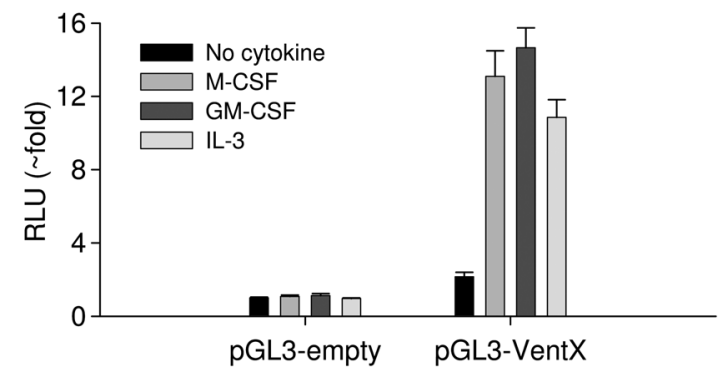

B
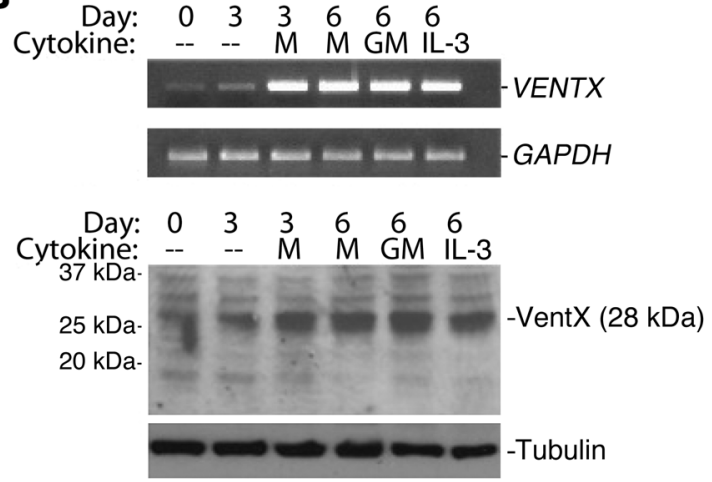

D

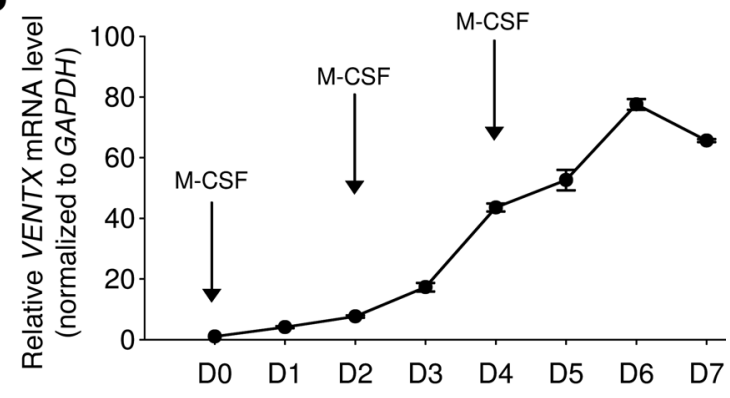

Figure 1

Upregulation of VentX expression during monocyte-to-macrophage differentiation. (A) VentX expression in circulating monocytes. Monocytes were magnetically isolated by anti-CD14 beads from peripheral blood of healthy adult donors. Total RNA was extracted, and RT-PCR analysis of VENTX mRNA level was conducted as described in Methods. (B) VentX expression during monocyte-to-macrophage differentiation in vitro. Monocytes were cultured in the presence of M-CSF (M), GM-CSF (GM), or IL-3 for the indicated days or in the absence of cytokines for 3 days. Upper panel: VENTX mRNA levels were determined by RT-PCR. Lower panel: VentX protein levels were determined by Western blot analysis, using VentX-specific antibodies. VentX expression in freshly isolated monocytes was used as baseline control (Day 0). (C) Induction of VENTX promoter activity by the indicated cytokines. Freshly isolated monocytes were electroporated with pGL3-VentX promoter luciferase reporter construct or a control empty pGL3 luciferase reporter. Twenty-four hours after electroporation, cells were treated with the indicated cytokines for an additional 24 hours. Cell lysates were then obtained, and luciferase activity was measured. Data represent mean + SD of triplicates of 1 representative experiment. (D) Time course of VentX expression during in vitro induction of monocyte differentiation into macrophages. Monocytes were subjected to M-CSF treatment in vitro for the indicated time. The VENTX mRNA levels were determined by quantitative PCR for up to 7 days.

expression in peripheral blood monocytes from 6 healthy donors by RT-PCR. We found that VentX expression was relatively constant among different individuals (Figure 1A). In comparison, VentX expression was upregulated during monocyte-to-macrophage differentiation induced by cytokines such as M-CSF, GM-CSF, and IL-3 (Figure 1B, upper panel). Using promoter luciferase assay, we found that VENTX promoter can be activated by the indicated cytokines (Figure $1 C$ ), suggesting that VentX expression was transcriptionally regulated by the differentiation signals. The regulated expression of VentX was further demonstrated by Western blot analysis, using VentX-specific antibody (Figure 1B, lower panel). The significant upregulation of VentX expression was largely dependent on the addition of the cytokines. Nevertheless, a slight but discernible upregulation of VentX expression was also observed in the absence of inducing factors (Figure $1 \mathrm{~B}$, lanes 1 and 2 ), which may reflect adhesion-induced spontaneous differentiation of monocytes. To quantitatively measure VentX expression during in vitro monocyte-to-macrophage differentiation, we performed a time course experiment with real-time PCR analysis. As shown in Figure 1D, VENTX expression was rapidly induced during the first 4 days of cytokine treatment and remained at a high level throughout the 7 days of in vitro culture.
Vent $X$ is required for human primary monocyte-to-macrophage differentiation. The increased expression of VentX during monocyte-tomacrophage differentiation suggests a potential role for VentX during the process. To address this hypothesis, we employed siRNA technology to knock down VENTX expression in primary monocytes. The efficacy of siVent $X$ was determined by quantitative PCR and Western blot analysis (Figure 2A). In vitro differentiation of peripheral blood monocytes to macrophages by M-CSF stimulation is accompanied by elongated or fibroblast-like morphology and the expression of cell surface CD71 antigen, a macrophagic differentiation marker (25-31). As shown in Figure 2B, knockdown of VENTX expression in monocytes abrogated the morphogenesis of the fibroblast-like shape (Figure $2 \mathrm{~B}$ ) and substantially diminished expression of the cell surface CD71 marker triggered by M-CSF treatment (Figure 2, C and D). Other macrophage phenotypic markers such as Fc $\gamma$ RI CD64, costimulatory molecules CD40 and CD86, and integrins CD11b and CD11c were also significantly downregulated in siVentX-transfected monocytes compared with siGFP-transfected control cells (Figure 3A). In support of its functional relevance in innate immunity, the expression of pattern recognition receptors such as TLR4, mannose receptor (MR), and 
A
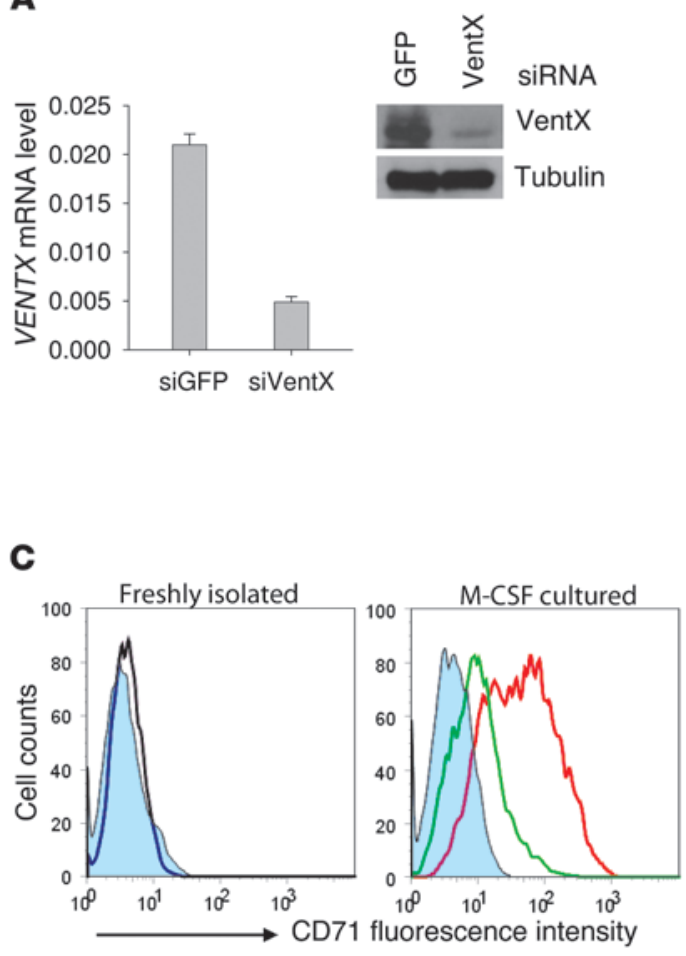

B
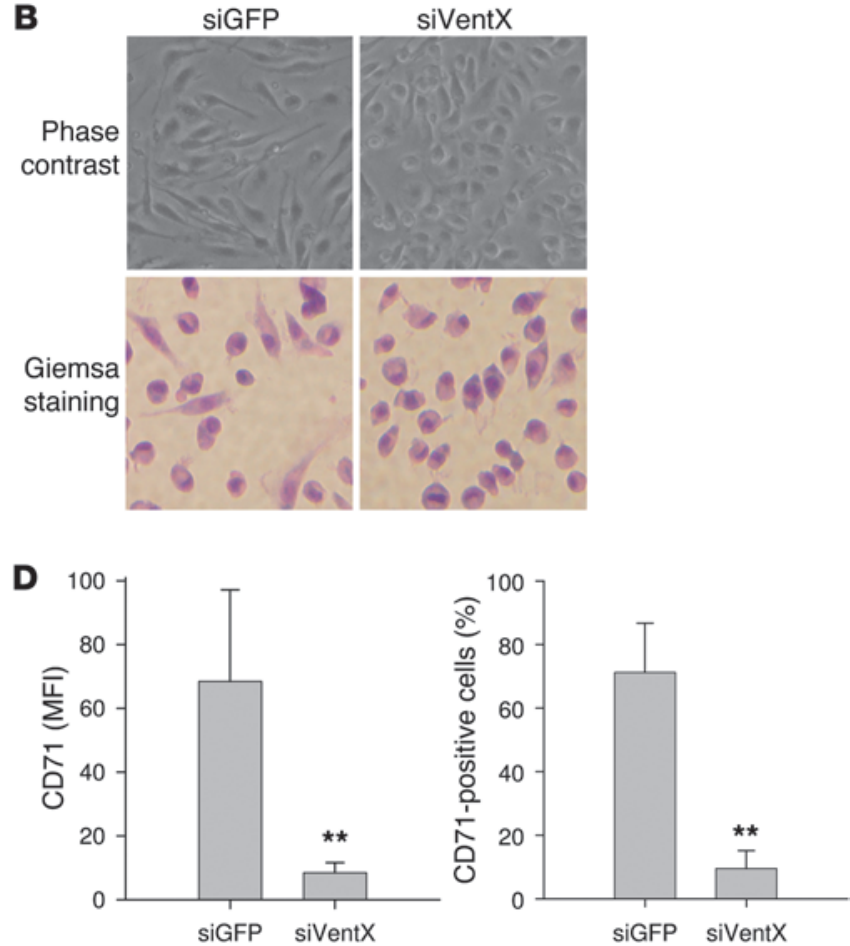

\section{Figure 2}

Knockdown of VENTX compromises the macrophage differentiation of primary monocytes. (A) Knockdown of VENTX expression in primary monocytes by RNA interference. Monocytes were transfected with siRNA against GFP or VentX through electroporation. VENTX mRNA levels were determined by real-time PCR at 3 days after transfection (left); VentX protein level was determined by Western blotting at 4 days after transfection (right). (B) Effects of VENTX knockdown on macrophage morphogenesis during M-CSF-induced differentiation. Monocytes were transfected with either siGFP or siVentX and subsequently exposed to $100 \mathrm{ng} / \mathrm{ml}$ M-CSF. At 4 days after transfection, the morphology of macrophages was revealed by phase contrast microscopy (upper panel) and Wright-Giemsa staining (lower panel). Original magnification, $\times 200$. Note: A portion of cells lost their original morphology during the Wright-Giemsa staining procedure. (C) Effects of VENTX knockdown on macrophage surface expression of CD71. Left panel: CD71 expression was not detected on cell surface of freshly isolated monocytes. Right panel: CD71 expression on M-CSF-treated monocytes at 4 days after siRNA transfection. Filled blue histogram represents the isotope control staining; red histogram represents monocytes transfected with siGFP; green histogram represents monocytes transfected with siVentX. (D) Bar graphs show mean + SD of 6 different experiments in $\mathbf{C}$. Paired $t$ test was used to reveal statistical significance. ${ }^{* \star} P<0.01$.

CD14 was also downregulated in VentX-silenced cells (Figure 3A). To eliminate the possibility of off-target effects, we used a second siRNA sequence (VentX siRNA-2) that has been shown to be effective in knockdown of VENTX expression (20). As shown in Supplemental Figure 1 (supplemental material available online with this article; doi:10.1172/JCI45556DS1), surface expression of CD71 and other markers was again downregulated by VentX siRNA-2 but not by a scrambled siRNA. It should be noted, however, that not all macrophage surface molecules are affected by the knockdown of VENTX. For example, expression of HLA-DR, CD16, and CD36 remained unchanged after VENTX knockdown (Supplemental Figure 2). Moreover, VENTX knockdown did not decrease the viability of primary monocytes as determined by propidium iodide (PI) and annexin $V$ staining assay (Figure $3 \mathrm{~B}$ ), therefore ruling out the possibility that diminished monocyte-to-macrophage differentiation may result from cytotoxicity induced by VentX suppression. The effect of VENTX knockdown on macrophage differentiation could be seen as early as 3 days after siRNA transfection and was still noticeable 6 days after transfection (Supplemental Figure 3). In addition to M-CSF, siVentX blocked macrophage differentiation by other known inducing factors, such as GM-CSF and IL-3 (refs.
25, 31, and Supplemental Figure 4). Monocyte-to-macrophage differentiation is associated with enhanced capability of phagocytosis (4). As shown in Figure 3C, in comparison with the control, monocytes transfected with siVentX displayed reduced phagocytotic activity, suggesting that VentX is required for functional development during monocyte-to-macrophage differentiation.

VentX promotes macrophage differentiation of U937 cells. U937 is a promonocytic cell line that has been widely used as a model to study monocyte-to-macrophage differentiation. It has been shown that ectopic expression of several transcription factors, such as BLIMP-1 and IRF-7, was able to trigger macrophage differentiation in $\mathrm{U} 937(17,18)$. To test whether VentX is a bona fide key regulator of monocyte-to-macrophage differentiation, we generated stable U937 cell lines expressing GFP or GFP.VentX under the control of doxycycline-inducible (DOX-inducible) promoter. As shown in Figure 4A, after 3 days of DOX induction, a large portion of U937 cells became GFP positive. In the GFP.VentX-transduced cells, more than $50 \%$ of GFP-positive cells acquired surface expression of CD11b, CD11c, and CD14, the markers of macrophage differentiation $(18,25,32)$, whereas few GFP-transduced cells acquired these markers (Figure 4A). Truncation analysis showed that 

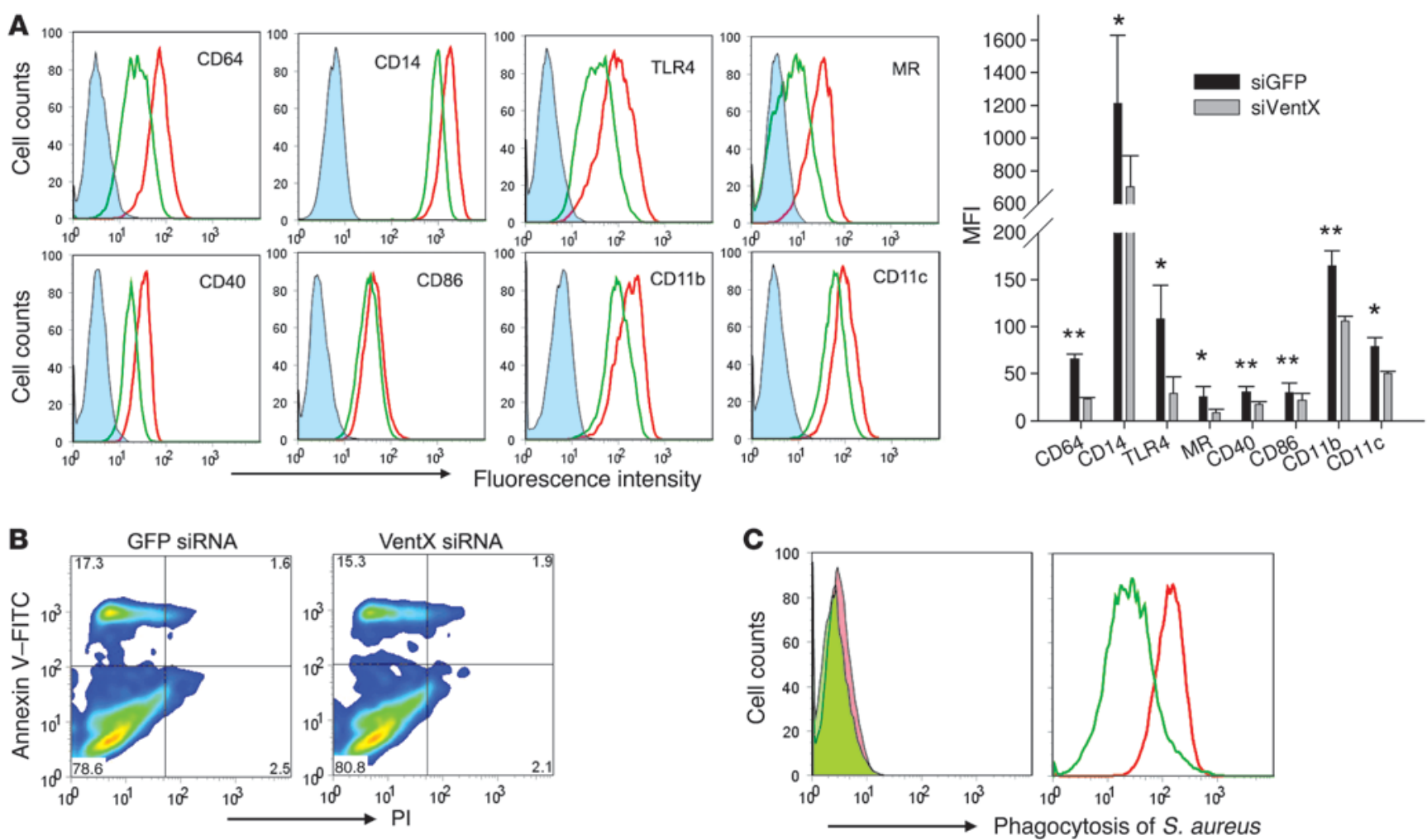

Figure 3

Effects of VENTX knockdown on surface markers expression, viability, and phagocytosis of macrophages. (A) Left panel: Flow cytometry analysis of indicated surface markers in monocytes transfected with siGFP or siVentX. Right panel: Bar graphs show the mean + SD of at least 3 separate experiments. ${ }^{*} P<0.05,{ }^{\star}{ }^{*} P<0.01$. (B) Effects of VENTX knockdown on macrophage viability. Monocytes were transfected with either siGFP or siVentX and subsequently exposed to $100 \mathrm{ng} / \mathrm{ml}$ M-CSF for 3 days. Cells were then harvested and stained with PI and annexin V and analyzed by flow cytometry. (C) Effects of VENTX knockdown on phagocytosis. Monocytes were transfected with siGFP or siVentX. Phagocytosis assays were carried out as described in Methods. Red histogram represents transfection with siGFP; green histogram represent transfection with siVentX. Left panel: Cells were incubated on ice. Right panel: Cells were incubated at $37^{\circ} \mathrm{C}$. Representative data of 3 independent experiments are shown.

the pro-differentiation function of VentX depends on its DNAbinding homeodomain (Supplemental Figure 5). In addition to the acquisition of surface markers, ectopic expression of VentX also led to pronounced morphological changes in U937 cells. As shown in Figure 4B, after 3 days of induction, GFP.VentX-transduced cells became adherent and flattened with extensive pseudopodia, resembling the morphology after ectopic expression of BLIMP-1 (18). In contrast, no such phenotypes were observed in U937 cells expressing GFP. Further, enhanced phagocytotic activity (Figure 5A) and increased expression (Figure 5B) and secretion (Figure 5C) of proinflammatory cytokines were also detected in GFP.VentX-transduced U937 cells, suggesting a role of VentX in the proinflammatory response of macrophages. Terminal macrophage differentiation of U937 cells is usually coupled with the cessation of cell division (18). Indeed, we observed that GFP. VentX-transduced $U 937$ cells were associated with apparent $G_{1}$ growth arrest (Figure 5, D and E), which is likely ascribed to the downregulation of $c-M y c$ and upregulation of $p 21$ after induction of VentX expression (Figure 5F).

Vent $X$ controls the expression of $M$-CSF receptor. Multiple signaling pathways and transcription factors have been implicated in monocyte differentiation (8). For identification of potential targets of VentX during monocyte differentiation, HL60 cells were ectopically expressed with VentX and treated with PMA to induce monocyt- ic differentiation (33). As shown in Supplemental Figure 6, VentX induced drastic upregulation of M-CSF receptor (M-CSFR) and transcription factors $M A F B$ and EGR1 in HL60 cells. No apparent changes were observed for C/EBP, AML1, IRF8, FOXP1, and $P U 1$, the master regulator of myeloid development (Supplemental Figure 6). Previous studies showed that M-CSFR is absolutely required for macrophage differentiation (34) and is a downstream target of multiple myeloid transcriptional factors; we therefore focused on the potential regulation of M-CSFR by VentX, using gain- and loss-of-function approaches. Overexpression of VentX in U937 cells resulted in induction of M-CSFR as determined by Western blot analysis of total cellular lysates (Figure 6A) and surface expression by flow cytometry analysis (Figure 6B). M-CSFR mRNA was also dramatically elevated as determined by real-time PCR (Figure 6C). To elaborate the mechanisms underlying VentXinduced M-CSFR expression, we examined the effects of VentX on $M$-CSFR transcription. Using $M$-CSFR promoter luciferase reporter assays, we observed that VentX significantly augmented $M$-CSFR promoter activity in U937 cells (Figure 6D, M-CSFR/WT). In comparison, VentX did not affect the activity of a control pGL3 luciferase reporter in this cell line. Detailed examination of the human $M$-CSFR promoter region revealed a putative homeodomain binding site (HDB) (Supplemental Figure 7A and ref. 35). Mutations of the HDB site in the $M$-CSFR promoter resulted in a significant 
A
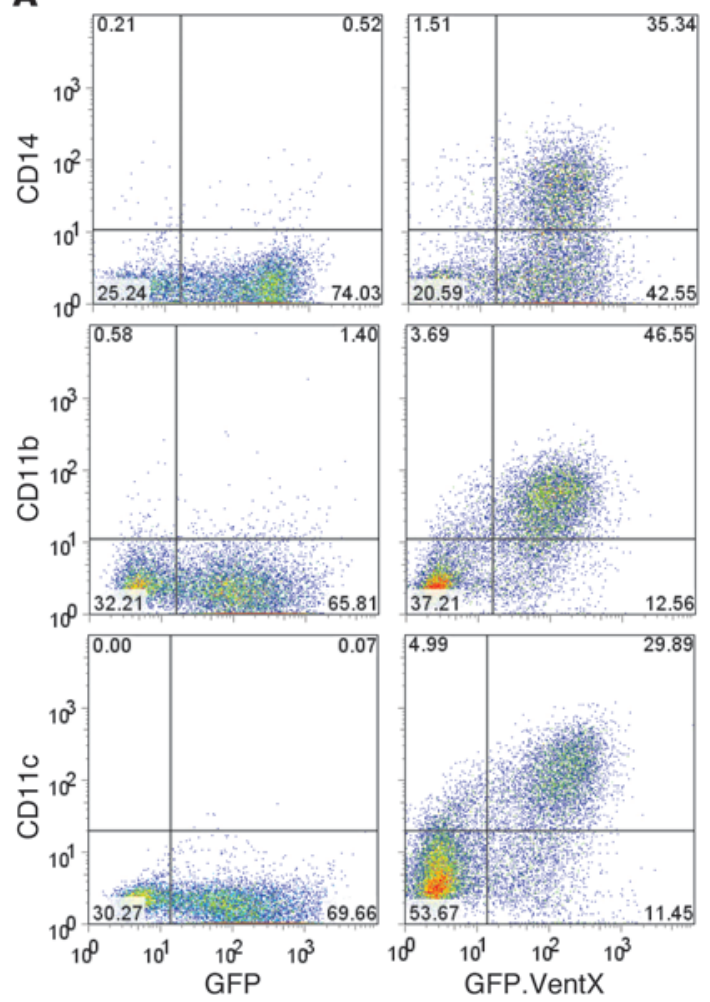
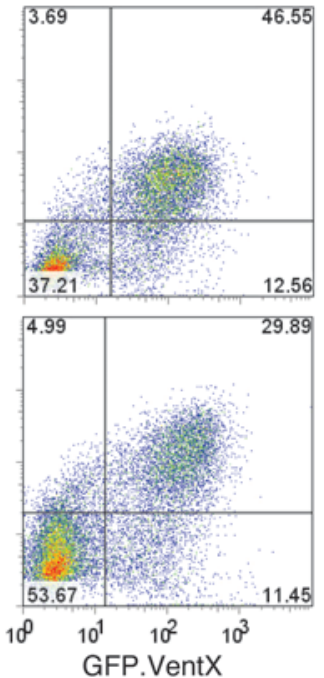

B

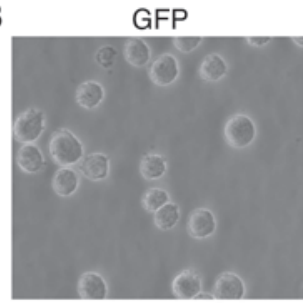

GFP

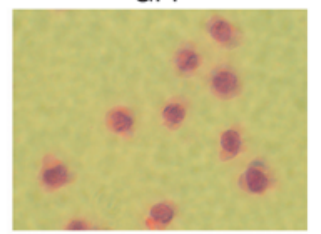

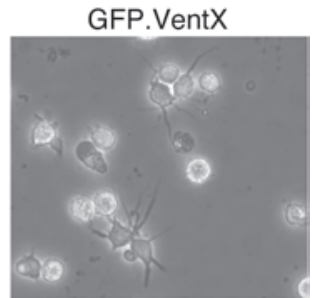

GFP.VentX

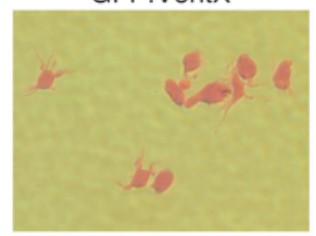

\section{Figure 4}

VentX promotes macrophage differentiation of U937 cells. U937 cell lines conditionally expressing GFP or GFP.VentX under the control of a tetracycline-inducible promoter were treated with $1.0 \mu \mathrm{g} / \mathrm{ml}$ DOX for 72 hours. (A) FACS analysis of monocyte/macrophage surface markers CD14, CD11b, and CD11c. (B) Effects of VentX expression on morphogenesis and adhesion of U937 cells. Upper panel: Cells were photographed using phase contrast microscopy. The percentage of adherent cells was calculated by methods of trypsinization and counting both floating and adherent fractions of cells: GFP, $<0.1 \%$; GFP.VentX, $35.3 \% \pm 5.0 \%$. Cells showing pseudopodia were counted as cells with morphological change: GFP, $<0.1 \%$; GFP.VentX, $28.9 \% \pm 3.7 \%$. Lower panel: Wright-Giemsa staining of DOX-treated U937 cells. Original magnification, $\times 200$.

decrease in VentX-induced luciferase activity, suggesting the functional importance of the HDB site (Figure 6D, M-CSFR/Mut). To determine whether VentX interacts with the M-CSFR promoter directly, we performed a ChIP assay and EMSA. The results of both tests suggested a direct interaction between VentX and the $M$-CSFR promoter (Figure 6, E and F). Moreover, the interaction between VentX and the M-CSFR promoter was largely abrogated by mutation of the HDB sites as revealed by the EMSA assay (Figure $6 \mathrm{~F}$, lane 3), suggesting the importance of the HDB in mediating interaction between VentX and the M-CSFR promoter. We then examined whether VentX is also required for M-CSFR expression in primary monocytes. To this end, we knocked down endogenous VENTX in primary monocytes by the siRNA method. In support of the overexpression experiments in the U937 cell model, knockdown of VENTX in primary monocytes caused significant downregulation of M-CSFR at mRNA, protein, as well as cell surface expression levels (Figure 7, A-C). To further assess the potential role of M-CSFR in mediating VentX function, we cotransfected M-CSFR plasmid with VentX siRNA into primary monocytes. As shown in Supplemental Figure 8, ectopic expression of M-CSFR partially restored the macrophage differentiation defect caused by VENTX knockdown, suggesting that VentX regulates monocyteto-macrophage differentiation, at least in part, through modulating the expression of M-CSFR.
Previous studies showed that the $M$-CSFR promoter is subjected to the regulation of multiple other transcriptional factors such as Foxp1, PU.1, AML1, and C/EBP (36). In particular, Foxp1 binds to the forkhead binding sites within the $M$-CSFR promoter and operates as a transcriptional repressor of M-CSFR expression (37), which raised the possibility that VentX may promote M-CSFR expression through displacement of Foxp1 occupancy on the M-CSFR promoter. To test this hypothesis, we performed ChIP assays to detect the interaction between Foxp1 and the $M$-CSFR promoter in U937 cells with ectopic expression of VentX and in primary monocytes with knockdown of VENTX. As shown in Figure 7D, neither overexpression nor knockdown of VENTX affected the binding of Foxp1 to the M-CSFR promoter, as shown by the ChIP assays. Notably, the binding site of VentX on the $M$-CSFR promoter is distant ( $450 \mathrm{bp})$ to that of Foxp1 (Supplemental Figure 7B).

Vent $X$ is required for the proinflammatory response in macrophages. Macrophages retain a high level of VentX expression (Figure 1, B and D). We therefore explored the requirement of VentX for macrophage functions. We found that, upon knockdown of VENTX expression, macrophages lost their adherence to a plastic surface and were not able to spread out and maintain their fibroblast-like shapes (Supplemental Figure 9A). The morphological changes of macrophages were associated with marked decreases in levels 
A

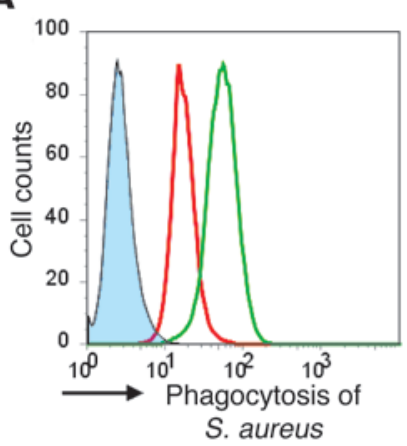

B

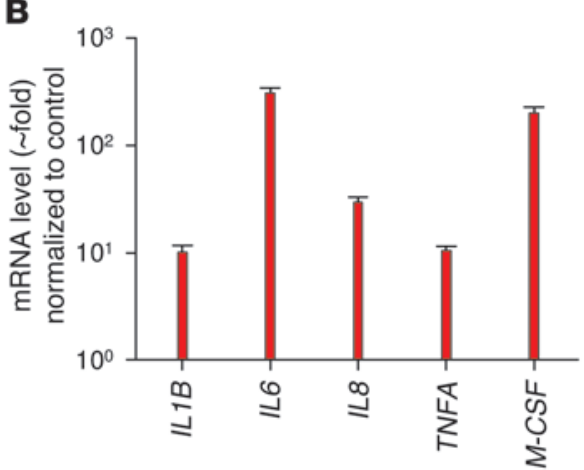

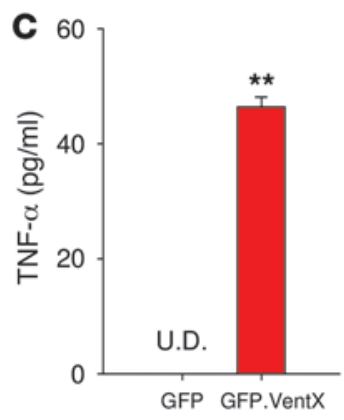

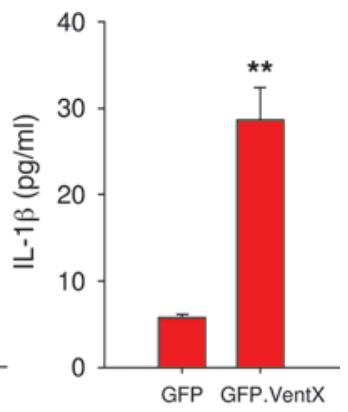

D

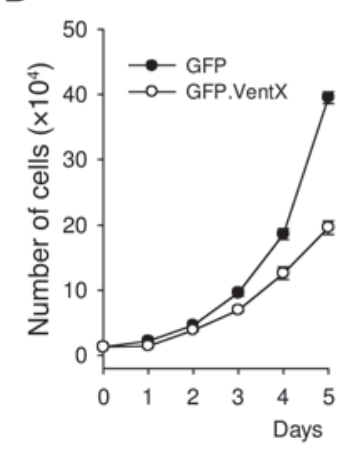

E

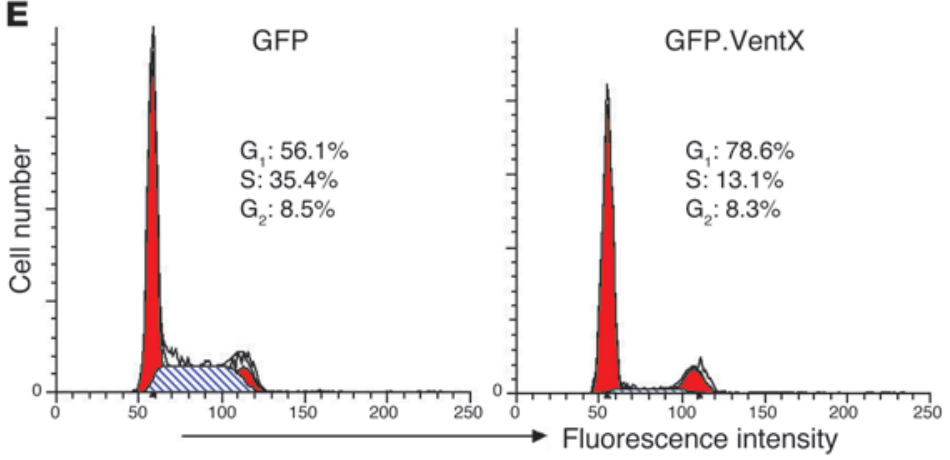

$\mathbf{F}$

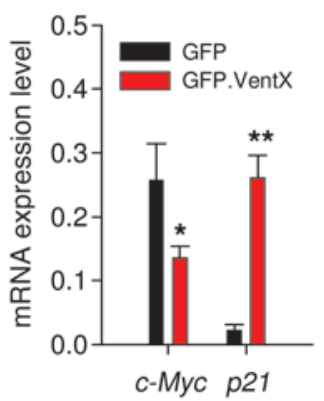

Figure 5

VentX promotes proinflammatory response and inhibits proliferation in U937 cells. U937 cell lines were treated as described in Figure 4. (A) Phagocytosis of DOX-treated U937 cells at $37^{\circ} \mathrm{C}$. Red histogram represent GFP-expressing cells; green histogram represent GFP.VentXexpressing cells. Filled blue histogram represents background staining of cells not undergoing phagocytosis. (B) Effects of VentX expression on the mRNA level of proinflammatory cytokines. U937 cells were treated with $1 \mu \mathrm{g} / \mathrm{ml}$ LPS for 6 hours after 72 hours exposure to DOX. Real-time PCR was performed to determine mRNA levels of the indicated cytokines. Data are presented as the fold of elevation and are mean + SD of triplicates from 1 representative experiment. (C) Secreted IL-1 $\beta$ and TNF- $\alpha$ from U937 cell culture supernatants were determined with ELISA kits. U.D., undetectable. Data represent mean + SD of triplicates from 1 representative experiment. (D) Effects of VentX on growth of U937 cells. Cells $\left(2 \times 10^{4}\right)$ were seeded in 6 -well plates and cultured for 5 days in the presence of $1.0 \mu \mathrm{g} / \mathrm{ml} \mathrm{DOX}$. Cell numbers at the indicated days were determined and plotted. (E) Cell cycle profiles of U937 cells expressing GFP or GFP.VentX after 3 days exposure to DOX. Cells were stained with PI and analyzed by FACS. (F) Effects of VentX on mRNA levels of $c-M y c$ and $p 21$ as determined by real-time PCR. Data represent mean $+\mathrm{SD}$ of triplicates from 1 representative experiment. ${ }^{\star} P<0.05$, ${ }^{\star \star} P<0.01$.

of cell surface adhesion molecules such as CD11b and CD11c, as determined by flow cytometry analysis (Supplemental Figure 9B), which may account for their aberrant morphologies (38).

Macrophages classically activated in vitro by IFN- $\gamma$ and LPS stimulation display elevated expression of MHC-II and costimulatory molecules CD80 and CD86 (2), secrete proinflammatory cytokines at a high level, and exhibit enhanced antimicrobial activity $(2,3)$. To investigate whether VentX is required for classical activation of macrophages, we used a loss-of-function approach involving knockdown of VENTX. First, we found that surface expression of CD40, CD80, and CD86, but not the HLA-DR, was significantly downregulated in macrophages transfected with siVentX (Figure $8 \mathrm{~A})$. Second, expression of proinflammatory cytokines such as TNF- $\alpha$, IL-1 $\beta$, IL- 6 , IL- 8 , and IL-12, as well as M-CSF, were significantly decreased in cells transfected with siVentX (Table 1). Consistently, there was a significant reduction in proinflammatory cytokine secretion by these siVentX-transfected macrophages (Figure 8B). Third, siVentX-transfected macrophages produced significantly less ROS (Figure 8C) and NO (Figure 8D) and displayed a weakened capacity to phagocytose (Figure 8E), suggesting that these cells are less effective in microbial killing. In addition, siVentX-transfected macrophages stimulated allogeneic $T$ cell pro- liferation at a lower rate, reflecting a decreased ability to present antigens (Figure 8F). Taken together, these experiments suggest that Vent $\mathrm{X}$ is required for the macrophage classical activation.

We also investigated whether VentX regulates the macrophage alternative activation following IL-4 treatment by analyzing several alternative activation markers such as CD163, MR, alternative macrophage activation-associated CC chemokine 1 (AMAC1; also called CCL18), and IL-10 $(6,39)$. As shown in Supplemental Figure 10 , although the level of CCL18 was downregulated, the expression of CD163, MR, and IL10 was not significantly affected by VENTX knockdown. Hence, VentX appears not to be essential for alternative activation of macrophages by IL-4.

VentX targets multiple pathways to regulate the proinflammatory response in macrophage. LPS and IFN- $\gamma$ are two key stimuli triggering the proinflammatory response of macrophages $(2,3)$. We therefore investigated whether VentX affected the signaling pathways of these two stimulants. We first examined whether VentX affected the expression of membrane receptors for LPS and IFN- $\gamma$. Using the U937 cell model, we found that ectopic expression of VentX significantly increased mRNA levels of the LPS receptor components (TLR4, CD14, MD-2) and IFN- $\gamma$ receptors (IFNGR1 and IFNGR2) (Figure 9A). Knockdown of VENTX 

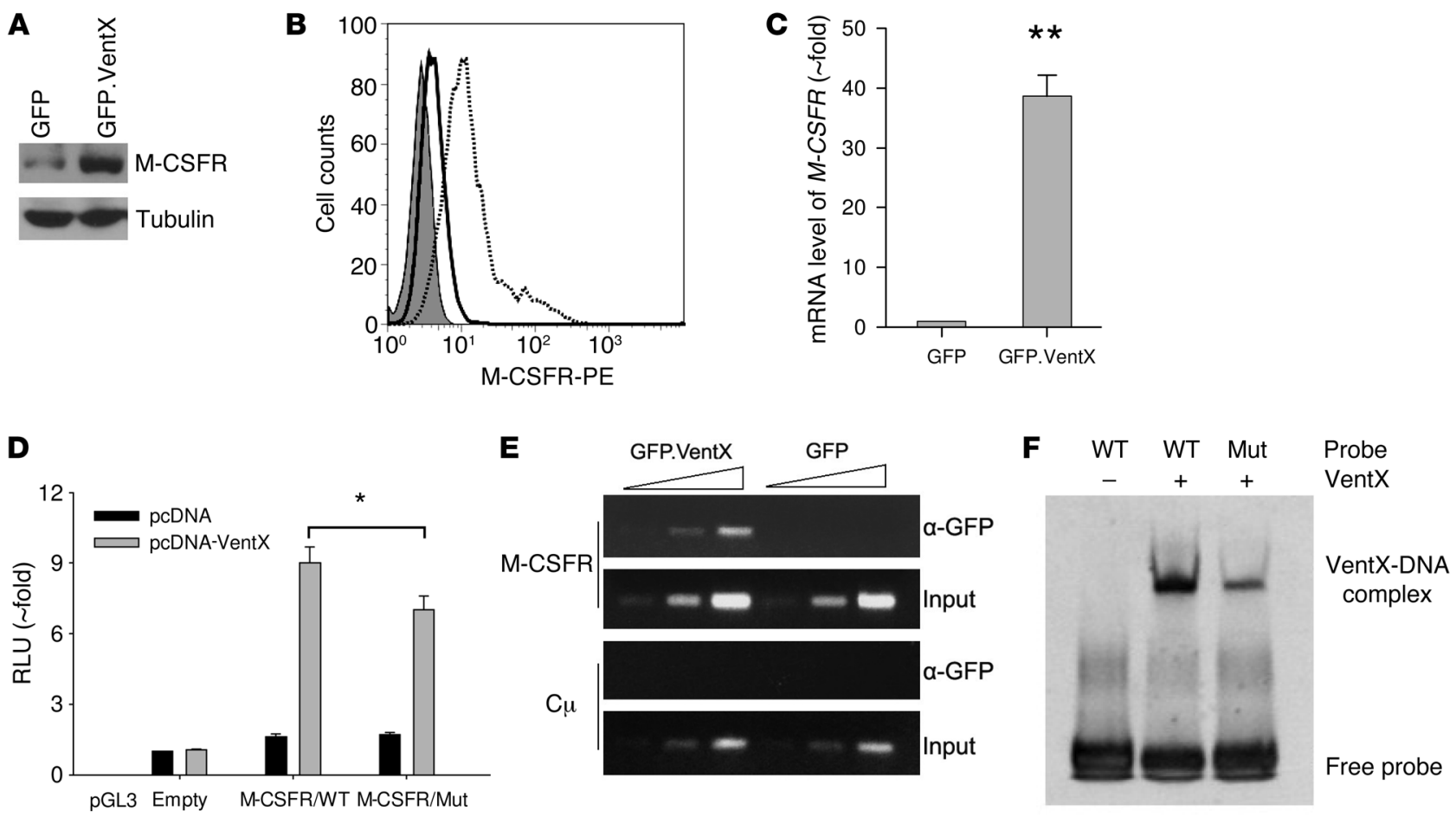

\section{Figure 6}

VentX transactivates M-CSFR expression. (A-C) U937 cell lines expressing GFP or GFP.VentX under the control of tetracycline-inducible promoter were treated with $1.0 \mu \mathrm{g} / \mathrm{ml}$ DOX for 72 hours. (A) Western blot analysis of M-CSFR protein levels from U937 cell lysates. Tubulin was used as a loading control. (B) Surface expression of M-CSFR was determined by FACS analysis. Filled gray histogram represents isotype control; solid line histogram represents cells expressing GFP; dotted line histogram represents cells expressing GFP.VentX. (C) M-CSFR mRNA levels were determined by real-time PCR. Data represent mean + SD of triplicates from 1 representative experiment. (D) VentX transactivation of $M$-CSFR promoter. pcDNA-VentX or pcDNA-control was cotransfected with wild-type or mutant $M$-CSFR promoter luciferase reporter constructs into U937 cells. The effect of VentX on M-CSFR promoter transactivation was determined by luciferase activity. Data are mean + SD of triplicates from 1 representative experiment. (E) ChIP analysis of the interaction between VentX and the $M$-CSFR promoter, showing the association of VentX with the M-CSFR promoter region but not with the C $\mu$ region in U937 cells. (F) Gel shift analysis showing the binding of VentX to the wildtype $M$-CSFR promoter probe, but reduced binding to the mutant $M$-CSFR promoter (Mut) probe. ${ }^{*} P<0.05,{ }^{* *} P<0.01$.

in primary macrophages consistently reduced the mRNA level of these receptors (Figure 9B). Flow cytometry analysis further confirmed that surface expression of CD119 (IFN- $\gamma$ receptor 1 ), CD14, and TLR4 was significantly diminished in siVentX-transfected macrophages (Figure 9C).

The TLR4 pathway is coupled to the activation of cytoplasmic transcription factors such as NF- $\mathrm{KB}$ and AP-1, which translocate to the nucleus and trigger profound changes in macrophage gene expression $(7,40)$. IFN- $\gamma$ signaling is largely mediated by the latent cytosolic factor Stat 1 that is activated by Jak kinase $(7,41,42)$. Thus, we examined whether VentX-targeted components of TLR 4 and IFN- $\gamma$ downstream signaling pathways. As shown in Figure 9, A and B, both gain-of-function experiments in U937 cells (Figure 9A) and loss-of-function experiments in primary macrophages (Figure 9B) demonstrated that VentX regulated the expression of multiple members of Jak/Stat, AP-1, and NF- $\mathrm{KB}$ signaling pathways. Particularly, mRNA levels of STAT1, JUNB, and C-FOS were substantially affected by the altered VentX expression (Figure 9, A and B). The mRNA data were further confirmed by Western blot analysis, which showed that the protein levels of Stat1, Jun-B, and c-Fos were subjected to modulation by VentX (Figure 9D). To explore the mecha- nisms whereby VentX regulates these factors, ChIP assays were performed but revealed no significant binding of VentX to the promoter regions of these genes (Figure 9E and data not shown), suggesting that VentX may regulate their expression indirectly.

Multiple other transcriptional factors have also been implicated in macrophage activation by previous studies. For example, Krüppel-like factor (KLF4) and HIF-1 are suggested to promote macrophage activation, whereas KLF2 and Stat 6 are inhibitory (43-48). In addition, emerging evidence has suggested the involvement of the transcriptional coactivator p300/CBP in the activation of macrophages $(43,44)$. We therefore examined whether VentX regulates the expression of these factors. As shown in Figure 10, while overexpression of VentX augmented mRNA levels of KLF2, KLF4, and HIF1 in U937 cells, knockdown of VENTX only caused downregulation of HIF1 mRNA in primary macrophages, suggesting that HIF-1 may be a physiological downstream target of VentX. VentX did not regulate the expression of $p 300$ mRNA (Figure 10). However, when $p 300$ was knocked down in U937 cells, VentX-induced upregulation of TNFA and IL1B became significantly compromised (Supplemental Figure 11), suggesting that p300 is involved in the VentXinduced proinflammatory response. 
A

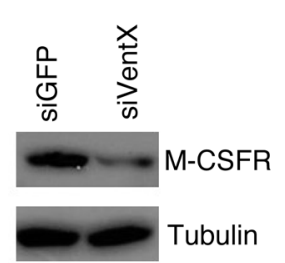

B

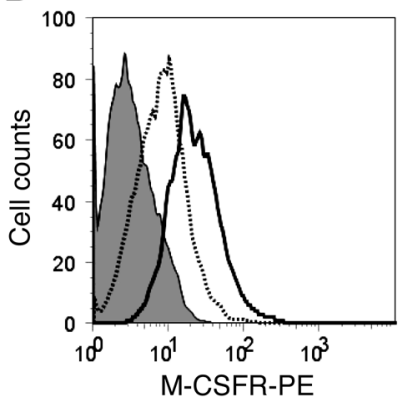

C

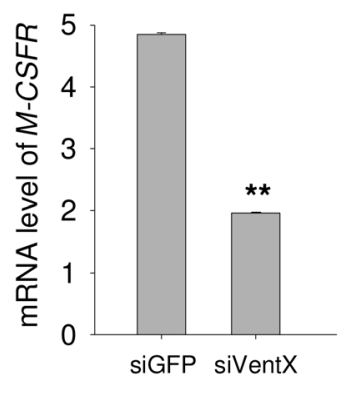

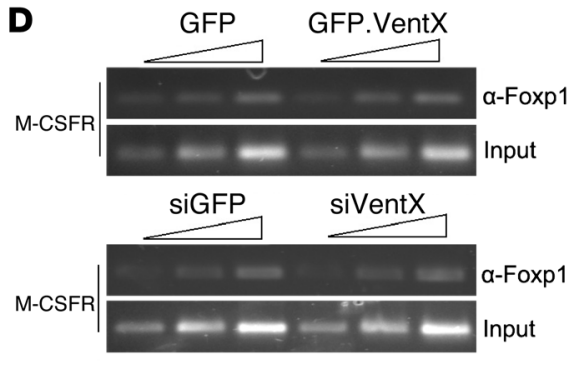

Figure 7

VentX regulates M-CSFR expression in primary monocytes. (A-C) Freshly isolated monocytes were transfected with siRNA against GFP or VentX. Cells were then maintained in RPMI 1640 medium supplied with M-CSF and harvested at 3 days after transfection. (A) Protein levels of M-CSFR in transfected cells were determined by Western blot analysis. (B) Surface expression of M-CSFR was determined by FACS analysis. Filled gray histogram represents isotype control; solid line histogram represents transfection with siGFP; dotted line histogram represents transfection with siVentX. (C) M-CSFR mRNA levels in transfected cells were determined by real-time PCR. Data represent mean + SD of triplicates of 1 representative experiment. (D) ChIP analysis of the effects of VentX on the interaction between Foxp1 and M-CSFR promoter in U937 cells (upper panel) and primary monocytes (lower panel) by overexpression (upper panel) and knockdown (lower panel) approaches. Note that VentX did not affect the interaction between Foxp1 and the M-CSFR promoter region. ${ }^{* \star} P<0.01$.

Expression of Vent $X$ and proinflammatory cytokines correlates in clinical patients. The findings that VentX controls proinflammatory responses in U937 cells (Figure 5) and primary macrophages (Figure 8) prompted us to determine the potential clinical relevance of these findings. We directed our exploration toward autoimmune diseases, such as SLE and RA. Previous studies have shown that expression of proinflammatory cytokines such as TNF- $\alpha$, IL- $1 \beta$, and IL- 6 is often elevated in these patients and that expression levels of proinflammatory cytokines are usually associated with disease severity (49-52). To assay for a potential relationship between VentX expression and the expression of proinflammatory cytokines in a clinical setting, we quantitatively measured the expression levels of TNFA, IL1B, IL6, and VENTX from peripheral blood leukocytes of SLE/RA patients. Linear regression analysis of the expression of TNF- $\alpha$, IL$1 \beta$, and IL- 6 against VentX was then performed. As shown in Figure $11 \mathrm{~A}, V E N T X$ expression strongly correlated with the expression of TNFA, IL1B, and IL6. Similar results were also obtained when purified monocytes from SLE/RA patients were used for the analysis (Figure 11B), suggesting a regulatory role of VentX in the expression of proinflammatory cytokines in SLE/RA patients.

Immunosuppressants remain a main therapeutic modality to control inflammation in SLE/RA patients. To determine whether VentX may serve as a therapeutic target of immunosuppressant treatment, we divided the SLE/RA patients into two subgroups: those on immunosuppressant and those on non-immunosuppressant regiments. As shown in Supplemental Figure 12, VentX expression was significantly downregulated in SLE/RA patients receiving immunosuppressants (treatment group) in comparison with that of the control group. Furthermore, expression levels of TNFA, IL1B, and IL6 were also decreased in patients receiving immunosuppressant treatment (Supplemental Figure 12).

\section{Discussion}

Vent $X$ regulates monocyte-to-macrophage terminal differentiation. The molecular mechanisms underlying monocyte/macrophage development have been extensively investigated for their broad implications in host defense, autoimmunity, inflammatory control, and tissue repair and regeneration $(1,3,10,53)$. Monocytes/macrophages arise from pluripotent hematopoietic stem cells in the bone marrow through multiple stages of concerted expression of signaling molecules and transcriptional factors $(8,10,11)$. Extensive genetic studies in knockout mice have identified several transcriptional factors that are important for specification of myeloid progenitor cells and subsequent monocyte/macrophage lineage commitment $(1,8,9,54)$. In particular, the Ets family transcriptional factor PU.1 and C/EBP family member $\mathrm{C} / \mathrm{EBP} \alpha$ represent master regulators of myeloid lineage development (11-14). Nevertheless, it is clear that monocyte/ macrophage lineage commitment is distinct from differentiation of monocytes into mature macrophages; the latter is accompanied by marked changes in cell morphology and immune functions and requires a new set of expressed genes $(15,16,38,53)$. However, little is known about the transcriptional regulation of terminal monocyteto-macrophage differentiation. Our data suggest that VentX is a key regulator of this process. In the knockdown experiments, 50\%-70\% of VENTX suppression could be achieved with the siRNA approach, which resulted in a remarkable $80 \%$ reduction in CD71 expression (Figure 2). Meanwhile, cells lost normal fibroblast-like morphology and showed weaker phagocytotic activity, indicating that VentX deficiency affects various aspects of macrophage differentiation and function. Moreover, consistent with the upregulated expression of VentX during monocyte-to-macrophage differentiation, overexpression of VentX in primary monocytes was able to promote macrophagic differentiation (our unpublished observations). Further, we showed that ectopic expression of VentX alone was sufficient to drive macrophagic differentiation of U937 cells. Although transcription factors, such as BLIMP-1, IRF-7, PU.1, and NF-кB, have been shown to be important for U937 differentiation, their specific involvement in primary human monocyte-to-macrophage differentiation was not defined $(17,18,55)$. To our knowledge, VentX is the first transcription factor both necessary and sufficient for human primary monocyte-to-macrophage differentiation. Interestingly, despite its prominent role in human monocyte-to-macrophage differentiation, we and others were unable to identify a murine homolog of VentX. Strikingly, the two genes that flank the VENTX gene in the human genome (UTF1 and ADAM8) are syntenic in the mouse genome; however, there appears to be no VENTX homolog in the mouse (24). 

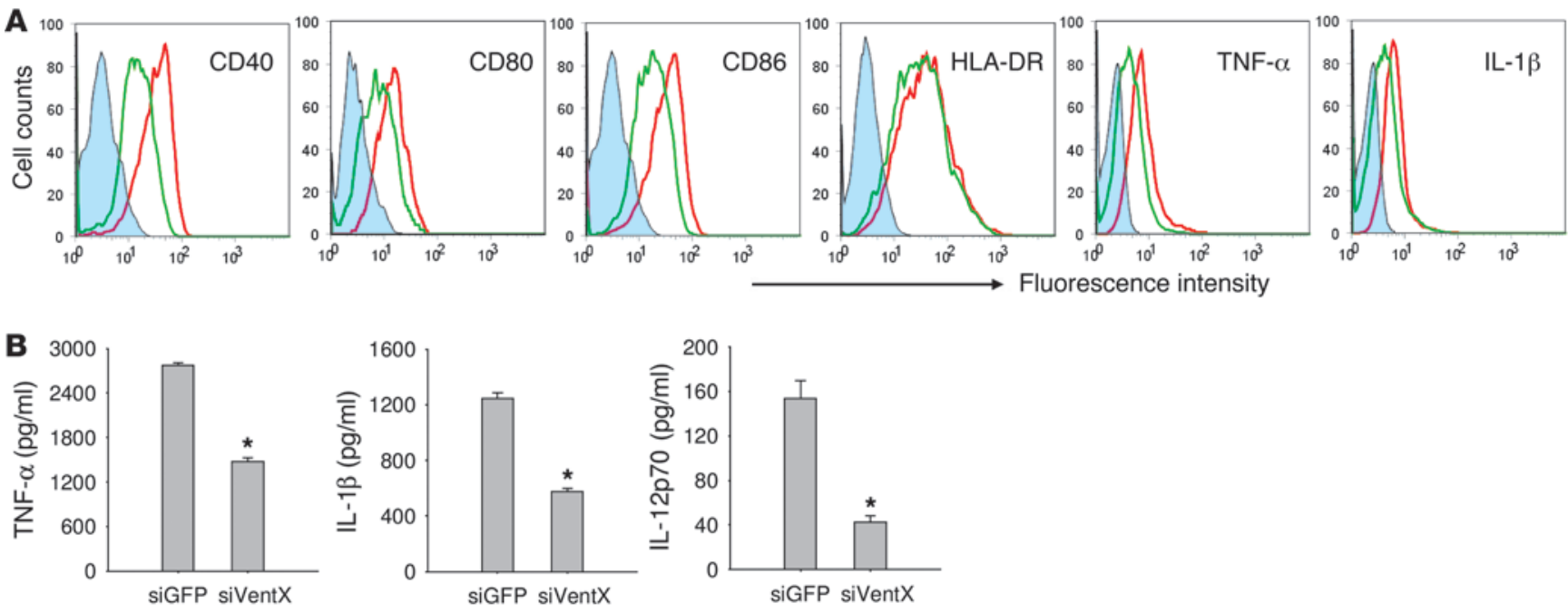

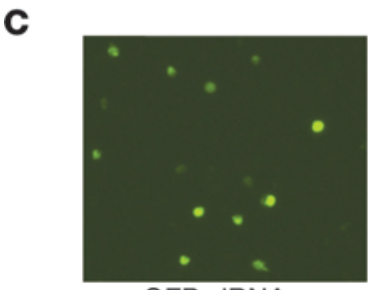

GFP siRNA

D

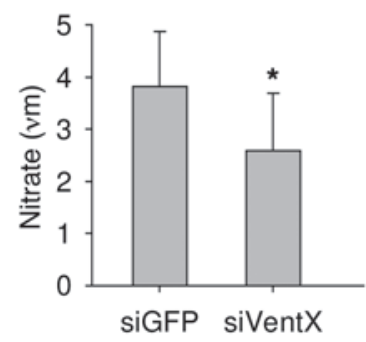

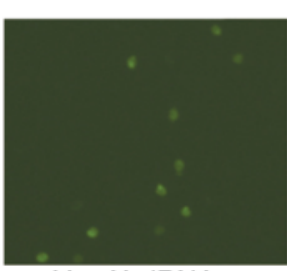

VentX siRNA

$\mathbf{E}$

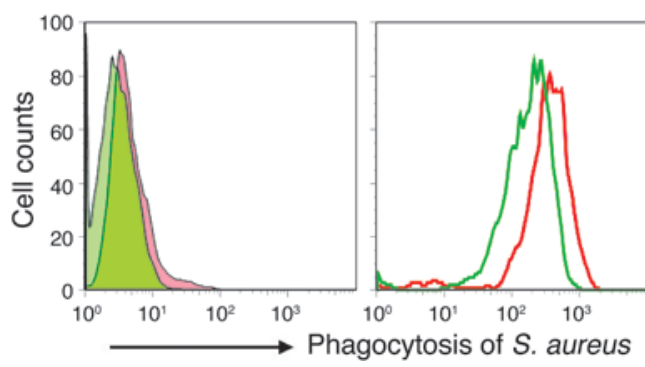

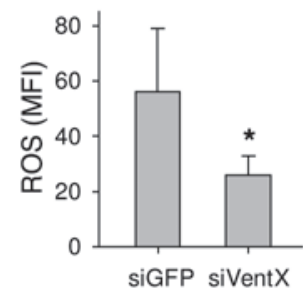

$\mathbf{F}$

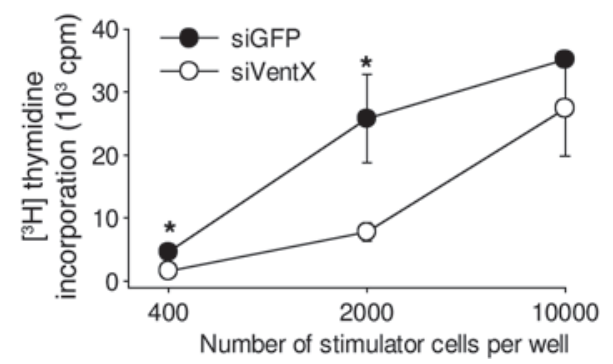

\section{Figure 8}

VentX expression is required for classical macrophage activation. Macrophages were generated through incubation of freshly isolated monocytes with $100 \mathrm{ng} / \mathrm{ml} \mathrm{M-CSF}$ for 4 days. These macrophages were then transfected with siGFP or siVentX and further cultured in RPMI 1640 medium for an additional 3 days. Thereafter, cells were exposed to $1 \mu \mathrm{g} / \mathrm{ml}$ LPS plus $20 \mathrm{ng} / \mathrm{ml}$ IFN- $\gamma$ for 6 hours. (A) Surface staining of CD40, CD80, CD86, and HLA-DR and intracellular staining of TNF- $\alpha$ and IL-1 $\beta$ were analyzed by flow cytometry. (B) Levels of secreted TNF- $\alpha$, IL-1 $\beta$, and IL-12p70 from culture supernatants were determined with ELISA kits. (C) ROS from siGFP-or siVentX-transfected macrophages was analyzed with fluorescence microscopy (left; original magnification, $\times 200)$ and flow cytometry (middle). Right panel: Mean + SD of 3 different flow cytometry experiments. (D) Nitrate level from siGFP- and siVentX-transfected macrophages. Data represent mean + SD of 3 different experiments. ${ }^{*} P<0.05$ (B-D). (E) Phagocytosis of siGFP- and siVentX-transfected macrophages. Red histogram represents transfection with siGFP; green histogram represents transfection with siVentX. Left panel: Cells were incubated on ice. Right panel: Cells were incubated at $37^{\circ} \mathrm{C}$. (F) Effects of VENTX knockdown on mixed lymphocyte reaction. Irradiated macrophages that had been transfected with siGFP or siVentX were utilized to stimulate allogenic naive CD4+ $T$ cell proliferation. The proliferation rates are presented as $\mathrm{cpm}$. Results show mean $+\mathrm{SD}$ of triplicate wells of 1 representative experiment. Statistically significant difference $\left({ }^{\star} P<0.05\right)$ was observed when $4 \times 10^{2}$ or $2 \times 10^{3}$ cells were added.

Among the questions to be answered are the molecular mechanisms of VentX upregulation during monocyte-to-macrophage differentiation (Figure 1). Promoter analysis with the ECR Browser (http://ecrbrowser.dcode.org/) found that the VENTX promoter region is highly conserved among primates (chimpanzee and rhesus monkey; Supplemental Figure 13A). Detailed analysis of the VENTX promoter revealed several evolutionarily conserved bind- ing sites for transcriptional factors implicated in macrophage differentiation, such as C/EBP, Egr-1, AP-1, and NF-кB (ref. 8 and Supplemental Figure 13B). Further studies are underway to define the role of these factors in the regulation of VentX expression.

Mechanism of regulation of macrophage differentiation by VentX. M-CSFR (also called csf1r) is an integral membrane tyrosine kinase encoded by the $c$-fms proto-oncogene. M-CSFR is 


\section{Table 1}

Effects of VENTX knockdown on cytokine mRNA expression in macrophages

\begin{tabular}{lccc}
\hline & sigFP & siVentX & $\boldsymbol{P}$ \\
TNFA & $0.46 \pm 0.04$ & $0.18 \pm 0.01$ & $<0.01$ \\
IL6 & $3.40 \pm 0.21$ & $1.78 \pm 0.14$ & $<0.01$ \\
M-CSF & $0.40 \pm 0.01$ & $0.14 \pm 0.01$ & $<0.01$ \\
IL12p35 & $1.77 \pm 0.21$ & $0.37 \pm 0.14$ & $<0.01$ \\
IL12p40 & $0.52 \pm 0.02$ & $0.61 \pm 0.05$ & $>0.05$ \\
IL8 & $471 \pm 10.2$ & $172 \pm 5.8$ & $<0.01$ \\
IL1B & $51.08 \pm 3.2$ & $21.93 \pm 1.8$ & $<0.01$ \\
& & & \\
\hline
\end{tabular}

expressed in monocytes/macrophages and their progenitors and is obligatory for macrophage differentiation $(56,57)$. Several cytokines and transcription factors regulate macrophage differentiation by modulating expression of M-CSFR $(37,58$, 59). As shown in Figure 6, we demonstrated that M-CSFR is a transcriptional target of VentX through various biochemical approaches. VentX binds to the HDB site of the M-CSFR promoter and directly transactivates M-CSFR expression. When M-CSFR plasmid was supplemented into siVentX-transfected monocytes, the macrophage differentiation defect was partially restored (Supplemental Figure 8), indicating that M-CSFR is a physiologically important target of VentX in macrophage differentiation. Interestingly, the promoter regions of human and murine $M$-CSFR genes show little conservation (analyzed by ECR Browser; data not shown). Therefore, transcriptional activation of M-CSFR by VentX is likely a unique pathway for human monocytes. Besides the M-CSFR pathway, the finding that ectopic expression of Vent $\mathrm{X}$ alone is able to drive macrophage differentiation of U937 cells suggests the existence of other mechanisms underlying VentX-induced macrophage differentiation, because M-CSF is not required for U937 differentiation. Previous studies have showed that downregulation of c-Myc and upregulation of $\mathrm{p} 21$ promote macrophage differentiation of U937 cells $(9,10,18,19,60)$. Our data show that c-Myc and $\mathrm{p} 21$ can be regulated by VentX in U937 cells (Figure $5 F)$. In addition, knockdown of VENTX also downregulated the expression of GM-CSF and IL-3 receptors (our unpublished observations), which may explain the differentiation defects after GM-CSF and IL-3 treatments (Supplemental Figure 4).

VentX controls macrophage proinflammatory responses. Knockdown of VENTX caused downregulation of multiple membrane receptors critical for innate and adaptive immunity, including TLR4, MR, Fcy receptor CD64, costimulatory molecules CD40, CD80, and CD86, and adhesion molecules CD11b and CD11c. As a consequence, the profoundly phenotypic and functional changes were observed in VentX-silenced monocytes/macrophages. Specifically, downregulation of TLR4, CD14, MR, and CD64 may contribute to the impaired phagocytotic ability of monocytes/macrophages (61). Aberrant morphogenesis and adherence may relate to the downregulation of adhesion molecules CD11b and CD11c (38). Downregulation of costimulatory molecules CD40, CD80, and CD86 on membrane may weaken the ability of macrophages to stimulate $\mathrm{T}$ cell proliferation (Figure 8F). VentX also regulates the expression of Stat 1 and AP- 1 transcription factors, the essential components of the IFN- $\gamma$ and LPS signaling pathways. Consequently, knockdown of VENTX rendered macrophages unable to mount an optimal proinflammatory response upon classical activation (Figure 8). In contrast, overexpression of VentX enhanced secretion of proinflammatory cytokines in U937 cells (Figure $5, \mathrm{~B}$ and $\mathrm{C}$ ). In addition, other factors implicated in macrophage activation, such as HIF-1 and p300 coactivator complex, may also be involved in VentX-mediated inflammatory response. Our study therefore indicates that VentX targets multiple pathways and plays critical roles in various aspects of monocyte/macrophage biology. The clinical implication of VentX in regulation of inflammatory response was suggested by the observation that expression levels of VentX and several proinflammatory cytokines correlate positively in patients with autoimmune diseases (SLE and RA). The pathogenesis of these diseases is multifactorial and involves abnormalities in both innate and adaptive immunity. The increased expression of proinflammatory cytokines such as TNF- $\alpha$ and IL- 6 has been related to the induction of the diseases $(62,63)$. Thus, dysregulated expression of VentX in macrophages may play a role in the pathogenesis of autoimmune diseases. Moreover, we found that expression of VentX could be downregulated by immunosuppressive therapy in SLE/RA patients (Supplemental Figure 12), suggesting a potential role of VentX as a future therapeutic target and in clinical management of autoimmune and inflammatory disorders.

\section{Methods}

Monocyte isolation and culture. PBMCs from healthy adult donors at Children's Hospital Boston were isolated by Ficoll density gradient centrifugation. Experiments with human materials were performed according to protocols approved by the institutional review committee of Brigham and Women's Hospital. CD14+ monocytes were purified from PBMCs using anti-CD14-coated microbeads (Miltenyi Biotec). The purity of freshly isolated $\mathrm{CD} 14^{+}$monocytes was more than $95 \%$ as analyzed by flow cytometry. Monocytes were cultured in 12 -well plates at $1 \times 10^{6} \mathrm{cells} / \mathrm{ml}$ with RPMI 1640 medium containing 10\% FBS. M-CSF, GM-CSF, and IL-3 were purchased from PeproTech and used at a final concentration of $100 \mathrm{ng} / \mathrm{ml}$. Cytokines were added to cultures every 2 or 3 days.

RNA interference. Human primary monocytes were transfected using the Human Monocyte Nucleofector Kit (Lonza) according to the manufacturer's instructions. Briefly, $5 \times 10^{6}$ monocytes were resuspended into $100 \mu \mathrm{l}$ Nucleofector solution with $0.5 \mathrm{nmol}$ of either VentX siRNA (forward: 5'-UUCAGAAUCGCCGCAUGAAACACAAACGG-3'; reverse: 5'-CCGUUUGUGUUUCAUGCGGCGAUUCUGAA-3') or non-effective GFP siRNA (forward: 5'-UGACCACCCUGACCUACGGCGUGCAGUGC-3'; 5'-reverse: GCACUGCACGCCGUAGGUCAGGGUGGUCA-3') before electroporation with the Nucleofector II Device (Lonza). Cells were then immediately removed from the device and incubated overnight with $1 \mathrm{ml}$ prewarmed Human Monocyte Nucleofector Medium containing $2 \mathrm{mM}$ glutamine and $10 \%$ FBS. Cells were then resuspended in complete RPMI medium and treated with appropriate cytokines to induce differentiation into macrophages. Similarly, macrophages derived from monocytes were transfected with Human Macrophage Nucleofector Kit (Lonza) following the manufacturer's instructions.

Generation of U937 cell line conditionally expressing VentX. Human promonocytic cell line U937 was obtained from ATCC. Plasmid constructs expressing VentX have been described previously (20). GFP.VentX fusion fragment was cut from pCS2 expression vector through digestion with BamHI/SnaBI and subcloned into pRetroX-Tight-Puro retroviral vector 

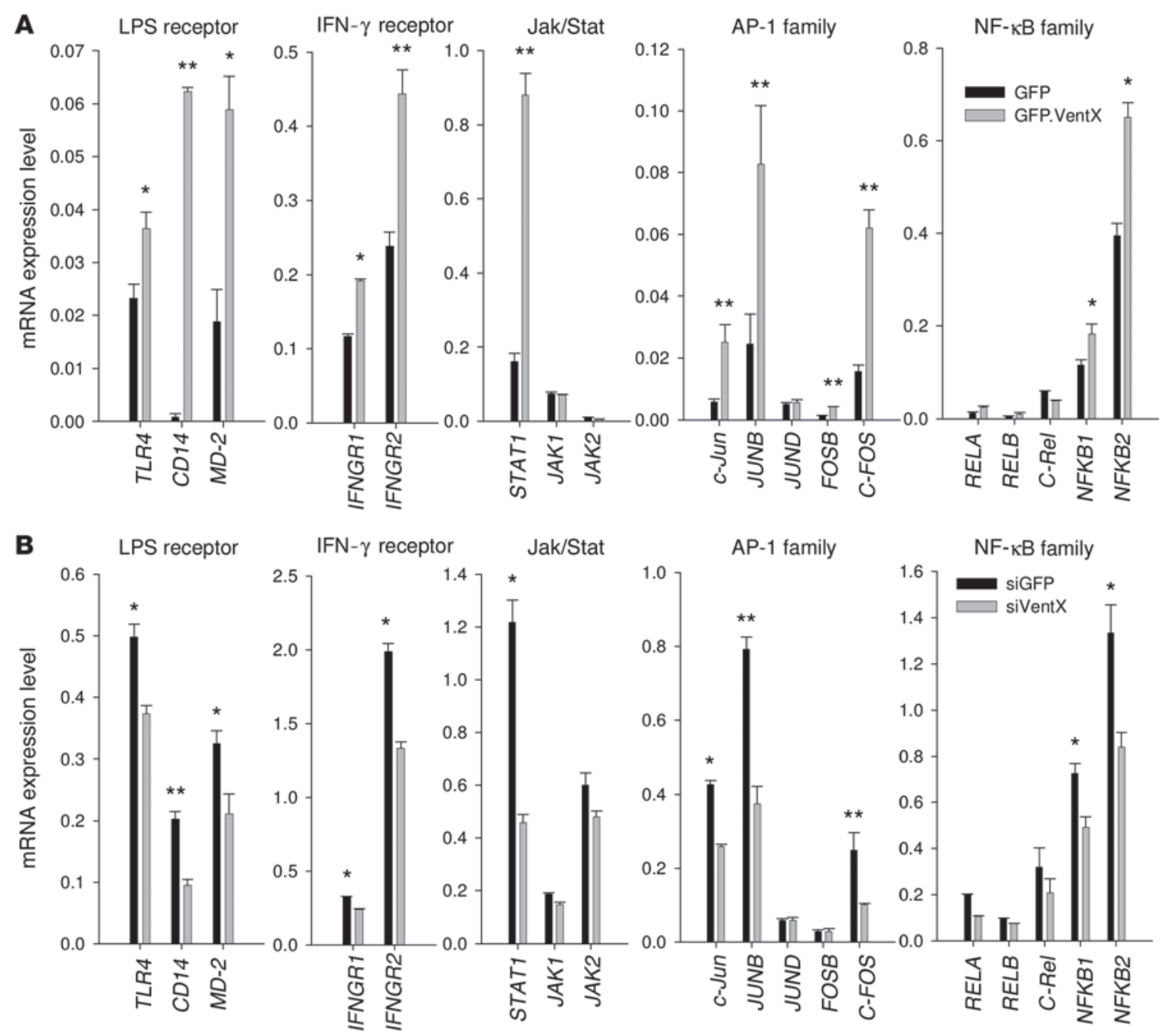

C

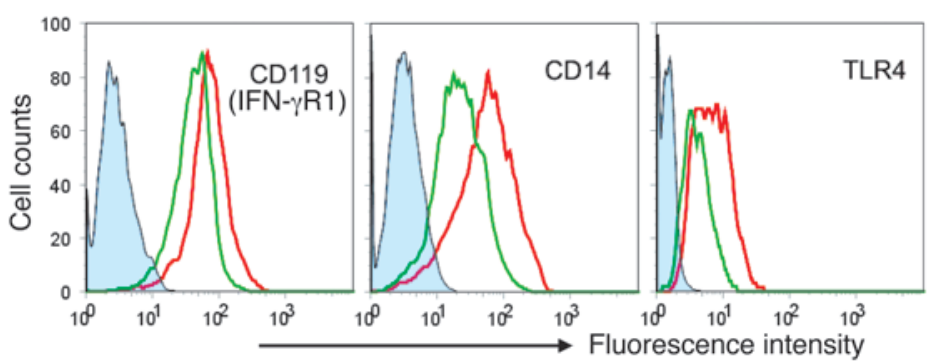

D

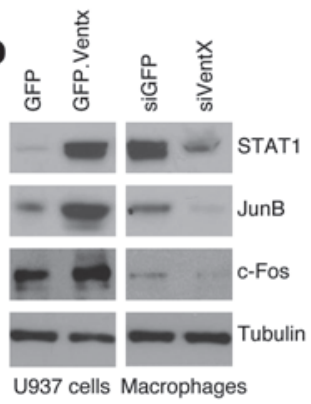

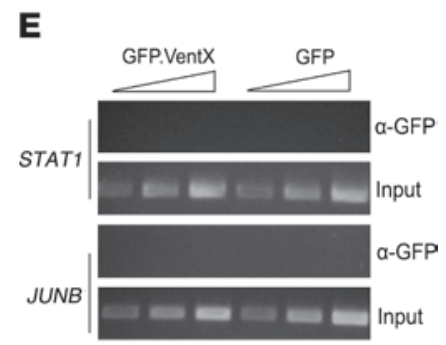

\section{Figure 9}

VentX regulates LPS and IFN- $\gamma$ signaling pathways. (A) Effects VentX overexpression on the expression of LPS and IFN- $\gamma$ pathway components. U937 cells expressing GFP or GFP.VentX under the control of a tetracycline-inducible promoter were treated with $1 \mu \mathrm{g} / \mathrm{ml}$ LPS for $6 \mathrm{hours}$ after exposure to DOX for 72 hours. Real-time PCR was performed to determine mRNA levels of the indicated genes. Data represent mean + SD of 2 separate experiments. (B) Effects of VENTX knockdown on the expression of LPS and IFN- $\gamma$ pathway components. Macrophages were transfected with siGFP or siVentX, followed by culture in RPMI 1640 medium for 3 days. Cells were then exposed to $1 \mu \mathrm{g} / \mathrm{ml} \mathrm{LPS}$ plus $20 \mathrm{ng} / \mathrm{ml}$ IFN- $\gamma$ for 6 hours. Real-time PCR was performed to determine mRNA levels of the indicated genes. Data represent mean + SD of triplicates of 1 representative experiment. (C) FACS analysis of surface expression of CD119, CD14, and TLR4 from macrophages transfected with siGFP or siVentX. Filled blue histogram represent the isotope control staining; red histogram represent macrophages transfected with siGFP; green histogram represent macrophages transfected with siVentX. (D) Western blot analysis of VentX effects on the protein level of STAT1, JunB, and c-Fos in U937 cells (left column) and macrophages (right column) by overexpression and knockdown approaches, respectively. (E) ChIP analysis of VentX interaction with STAT1 and JUNB promoter regions. No significant binding of VentX to the promoter regions of STAT1 and JUNB was detected by the ChIP assay. ${ }^{*} P<0.05,{ }^{* *} P<0.01$. 

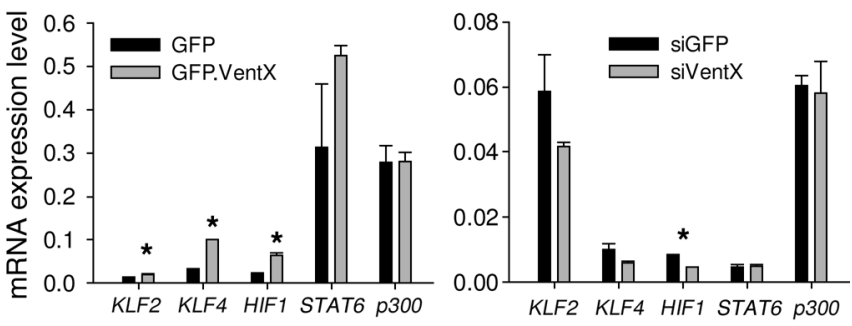

(Retro-X Tet-On Advanced Expression System, Clontech) digested with BamHI/NruI. Retroviruses were packaged through co-transfection of pCL-Ampho packaging vector (IMGENEX) and retroviral vectors into HEK293 cells. A U937 cell line conditionally expressing GFP.VentX was generated through co-transduction of pRetroX-GFP.VentX and pRetroXTet-On Advanced retroviruses. GFP.VentX-positive cells were sorted by a FACSAria high-speed sorter (BD) after incubation with $1.0 \mu \mathrm{g} / \mathrm{ml}$ DOX for 24 hours (Dana-Farber Cancer Institute Flow Cytometry Core Facility). Sorted cells were then maintained in RPMI 1640 medium in the absence of DOX. A U937 cell line conditionally expressing GFP was similarly generated for comparison.

FACS analysis. Phenotypic analysis of monocytes/macrophages was performed using flow cytometry after immunolabeling of cells with fluorescence dye-conjugated antibodies. The following antibodies were used: PEconjugated anti-CD71, -CD11b, -CD11c, -CD16, -CD64, -CD80, -CD86, -HLA-DR, -CD14, -TLR4, -IL-1 $\beta$, and -TNF- $\alpha$, and FITC-conjugated antiCD40 and -CD36 (eBioscience); FITC-conjugated anti-MR and unconjugated mouse anti-M-CSFR (R\&D Systems). Isotope control labeling was performed in parallel. Antibodies were diluted as recommended by the supplier. PE-conjugated rabbit anti-mouse IgG antibody was used for secondary M-CSFR staining. Labeled cells were analyzed with a FACScan flow cytometer (BD) using CellQuest software (BD). Results are expressed as the percentage of positive cells and/or MFI values after subtraction of the MFI obtained with the isotype control antibody.

Western blot analysis. Cells were lysed in solution A $(50 \mathrm{mM}$ Tris- $\mathrm{HCl}$, pH 7.8, $420 \mathrm{mM} \mathrm{NaCl}, 1 \mathrm{mM}$ EDTA, 0.5\% Nonidet P-40, $0.34 \mathrm{M}$ sucrose, $10 \%$ glycerol, $1 \mathrm{mM} \mathrm{Na}_{3} \mathrm{VO}_{4}, 10 \mathrm{mM} \mathrm{NaF}$ and $\beta$-glycerophosphate, $1 \mathrm{mM}$ PMSF, and protease inhibitor cocktail) as described previously (64). Proteins resolved by SDS-PAGE were transferred onto a PVDF membrane and were detected with optimal dilutions of primary antibodies, followed by HRP-linked secondary antibodies. Primary antibodies used were from Cell Signaling Technology, except anti-VentX serum, which was generated in our laboratory.

RT-PCR. Total RNA was isolated by the TRIzol reagent, and an equal amount of RNA was used for first-strand cDNA synthesis with SuperScript III First-Strand Synthesis System (Invitrogen) according to the manufacturer's protocol. To amplify VentX cDNA with conventional PCR, we used the AccuPrime Taq DNA polymerase system (Invitrogen) following the manufacturer's instructions. PCR products were separated on $2 \%$ agarose gels and stained with ethidium bromide. GAPDH was used as an internal control. We performed quantitative measurement of VentX and cytokine cDNA with SYBR Green on a LightCycler (480 Real-Time PCR System; Roche). The primers used are listed in Supplemental Table 1.

Cytokine measurements. Levels of IL-1 $\beta$ and TNF- $\alpha$ and IL-12p70 in the supernatants of E. coli LPS- (Sigma-Aldrich) and IFN- $\gamma$ - treated (PeproTech) macrophage or LPS-treated U937 cells were quantified using ELISA kits obtained from eBiosciences. Analyses were conducted according to the manufacturer's instructions.

\section{Figure 10}

Effects of VentX on the mRNA level of additional genes implicated in macrophage activation. VentX expression in U937 cells and macrophages was modulated as described in Figure 9. The mRNA levels of indicated genes were determined by real-time PCR. ${ }^{*} P<0.05$.
Phagocytosis assay. Phagocytosis of cultured monocytes/macrophages or U937 cells was performed with pHrodo Staphylococcus aureus BioParticles conjugates from Invitrogen. Briefly, S. aureus particles were first sonicated to achieve a homogeneous solution and then opsonized for 60 minutes at $37^{\circ} \mathrm{C}$ with opsonizing reagent obtained from Invitrogen (catalog S-2860). After the S. aureus particles were washed 3 times in PBS, they were resuspended to an appropriate concentration for phagocytosis assay. Cells $\left(5 \times 10^{5}\right)$ were incubated with $100 \mu$ l opsonized particles for 2 hours at $37^{\circ} \mathrm{C}$. After extensive washings, cellular phagocytosis of bioparticles was monitored by flow cytometry. Negative controls were also performed in parallel by incubating cells with particles on ice instead of at $37^{\circ} \mathrm{C}$.

Detection of ROS and NO. The ROS level in activated macrophages was detected with Image-iT LIVE Green Reactive Oxygen Species Detection Kit (Invitrogen) basically following the manufacturer's instructions, except that the results were analyzed by both fluorescence microscopy and flow cytometry. The NO level was determined by Griess Reagent Kit for Nitrite Determination (Invitrogen) following the protocol provided by the manufacturer.

Cytostaining. For Wright-Giemsa staining, a staining kit from SigmaAldrich was used according to the manufacturer's instructions.

Mixed lymphocyte reaction. Macrophages were generated by incubating CD14-beads isolated monocytes with $100 \mathrm{ng} / \mathrm{ml} \mathrm{M-CSF}$ for 4 days. Macrophages were then transfected with siRNA against VentX or GFP, as described above. After 3 days of transfection, the cells were harvested, washed 3 times in PBS, and irradiated (50 Gy) before incubation with allogenic naive $\mathrm{CD}^{+} \mathrm{T}$ cells $\left(10^{5} /\right.$ well) for 7 days in 96 -well flat-bottom microplates (Costar). Various numbers of irradiated macrophages were added as indicated. The cells were pulsed for the last 18 hours with $1 \mu \mathrm{Ci}$ $\left[{ }^{3} \mathrm{H}\right]$ thymidine to determine $\mathrm{T}$ cell proliferation.

Luciferase reporter assay. The $1.56-\mathrm{kb}$ fragment of the M-CSFR promoter region was amplified with forward primer: $5^{\prime}$-GTTACGCGTGGGAAGCCAAGGTATGAGAATC-3'; and reverse primer: 5'-AAGCTCGAGCCTCGGTGGGGAAGTGGCAG-3'. The 2.8-kb fragment of the VENTX promoter region was amplified with forward primer: 5 '-CAGCCGAGTCTCACTCTGTC-3'; and reverse primer: 5'-CAAAGCTGGAGAGCTGCTGC- $3^{\prime}$. The PCR product was subsequently cloned into pGL3 luciferase reporter. $500 \mathrm{ng}$ reporter plasmid with $500 \mathrm{ng}$ of pcDNAVentX plasmid or empty pcDNA vector were transfected into U937 cells or primary monocytes through electroporation. Renilla luciferase plasmid (10 ng) was included for each transfection to normalize reporter activity. Cells were harvested at 48 hours after transfection and analyzed with Dual-Luciferase Reporter Assay System (Promega).

Gel shift assay. VentX protein was generated with a in vitro translation kit (TNT Coupled Reticulocyte Lysate Systems, Promega). The pCS2VentX plasmid with SP6 promoter was used as template, and translated VentX protein was verified by Western blot analysis. Gel shift assay was performed with a fluorescence-based EMSA Kit from Invitrogen following the manufacturer's instruction. The following double-stranded 
A
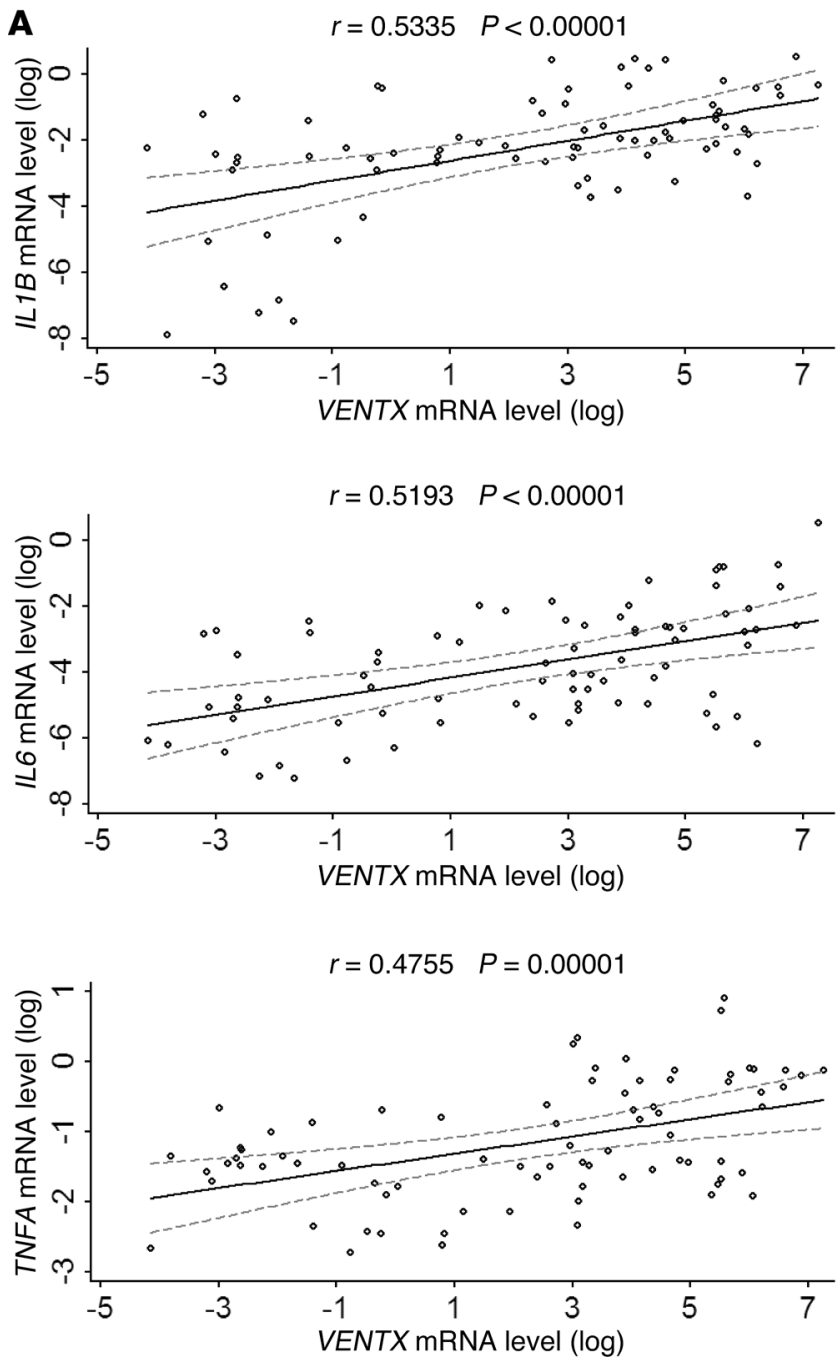
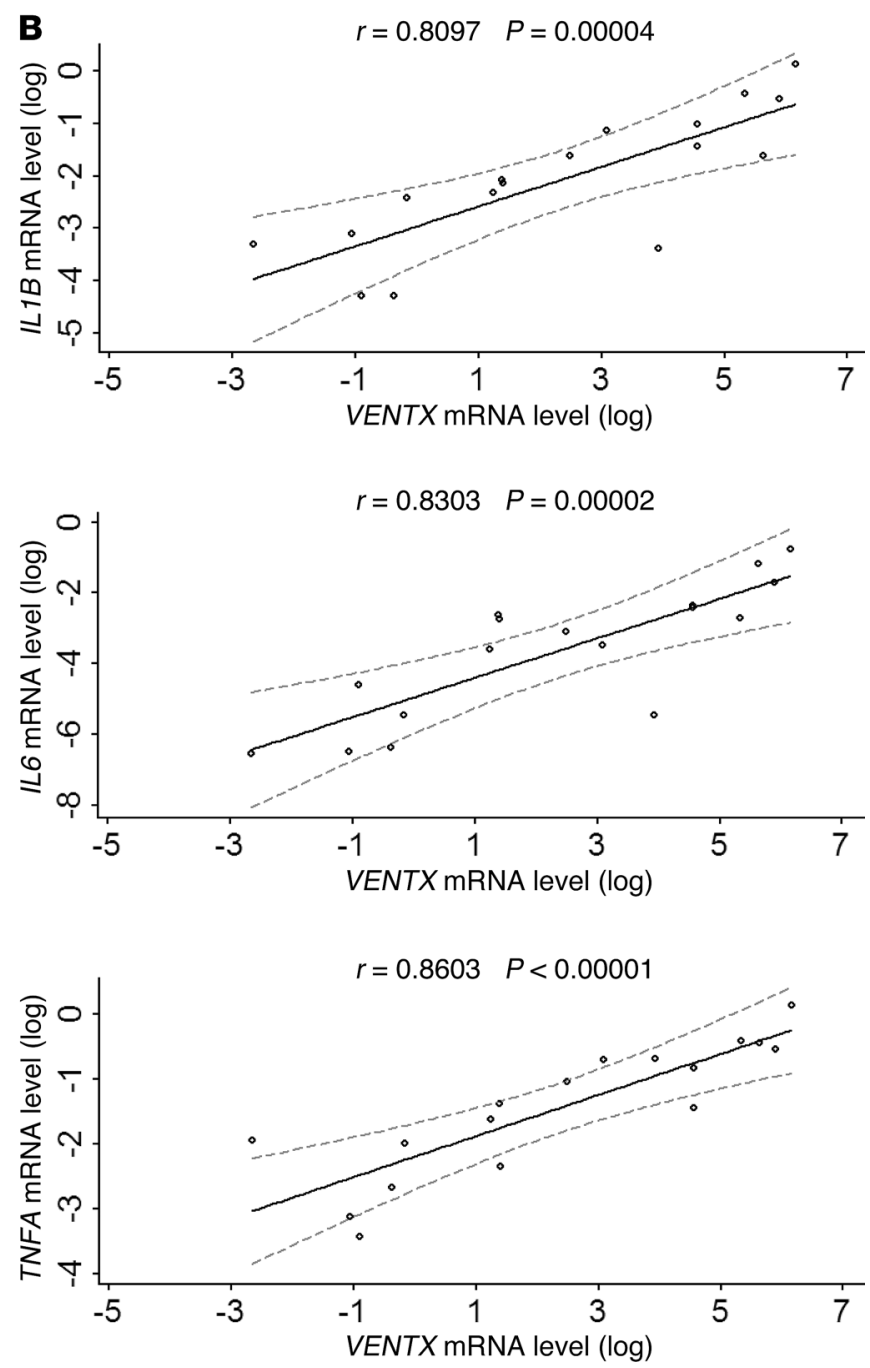

Figure 11

VentX expression correlates with IL1B, IL6, and TNFA levels in SLE/RA patients. (A) Total RNA was isolated from peripheral blood leukocytes of SLE/RA patients. Quantitative measurements of mRNA levels of VENTX and the indicated cytokines were performed as described in Methods. Scatter distributions and regression lines (solid lines) of the mRNA levels of $I L 1 B$, IL6, and TNFA were plotted against VENTX mRNA level from all patients. Confidence intervals of $99 \%$ are indicated by the dashed arcs. The regression coefficients $(r)$ were highly statistically significant as indicated. (B) Total RNA was purified from peripheral monocytes of SLE/RA patients. Scatter distributions and regression lines were plotted as above.

oligonucleotides were used in the experiments: 5'-CTGCGTCTCTAAA ATAATAATAATAAATTTTTAAAAGATATGC-3' (wild-type M-CSFR probe, putative homeodomain binding sequence is in bold and underlined); 5'-CTGCGTCTCTAAAATAAGAAGAATAAATTTTTAAAAGATATGC-3' (mutant M-CSFR).

ChIP assay. U937 cell lines conditionally expressing GFP or GFP.VentX were employed to detect the potential interaction of VentX with M-CSFR, STAT1, and JUNB promoters. Cells were treated with $1.0 \mu \mathrm{g} / \mathrm{ml}$ DOX for 2 days and harvested for ChIP assay. The ChIP procedure was performed with a kit from Upstate Biotechnology following the manufacturer's instructions. The GFP antibody (Santa Cruz Biotechnology Inc.) was used for the immunoprecipitation. The $M$-CSFR promoter region containing a putative HDB was amplified with specific primers $5^{\prime}$-TAGAGATAACGTCAGATCTCAC- 3 ' and $5^{\prime}$-CAAAGAGAAGTTAGGTTGCATG- ${ }^{\prime}$; the STAT1 promoter was amplified with primers $5^{\prime}$-TGACTGATGGAAAGGGGTGG-3' and 5'-GCCACCTGTTCTTGGGAGAT-3'; the JUNB promoter was amplified with primers 5'-GCTTACTAGCTTTCTGCATA-3' and 5'-GGAGGGGAGAGATCAAAAGG-3'; the constant region of immunoglobulin $M$ heavy chain gene $(\mathrm{C} \mu)$, which served as a negative control, was amplified with the following primers: 5'-AACCCTTTTCCCCCTCGTCT-3' and 5'-AGCACCTGTGAGGTGGCTGC- ${ }^{\prime}$. To detect whether VentX competes with Foxp1 to bind to the M-CSFR promoter region, U937 cells were treated with $1.0 \mu \mathrm{g} / \mathrm{ml} \mathrm{DOX}$ for 2 days as described above, or primary monocytes were transfected by electroporation with siGFP or siVentX. Cell lysates were then immunoprecipitated with Foxp1 antibody (Cell Signaling Technology), and the $M$-CSFR promoter region containing the Foxp 1 binding site was amplified with primers $5^{\prime}$-GCTTTAGAAGGGCCCCAAAC-3' and 5'-CTACTAGCTCCGCAGGGATC-3'. All PCR products were separated on $8 \%$ polyacrylamide gel and visualized by ethidium bromide staining.

Statistics. Data were analyzed using paired Student's $t$ test (2-tailed) and Wilcoxon rank-sum test. Differences with $P$ value less than 0.05 were considered statistically significant. 


\section{Acknowledgments}

We thank Marc Kirschner for critical input; D. Cohen and R. Blumberg for critical reagents and use of their facilities; Yinke Yang and Edward W. Zhou for technical support; and M. Freeman for critical reading of the manuscript. $Z$. Zhu was supported by grants from the NIH, Department of Defense, and American Cancer Society and research funds from Brigham and Women's Hospital.
Received for publication October 26, 2010, and accepted in revised form April 27, 2011.

Address correspondence to: Zhenglun Zhu, Department of Medicine, Brigham and Women's Hospital, Boston, Massachusetts 02115, USA. Phone: 617.732.5467; Fax: 617.730.5807; E-mail: zzhu@partners.org.
1. Auffray C, Sieweke MH, Geissmann F. Blood monocytes: development, heterogeneity, and relationship with dendritic cells. Annu Rev Immunol. 2009;27:669-692.

2. Martinez FO, Sica A, Mantovani A, Locati M. Macrophage activation and polarization. Front Biosci. 2008;13:453-461.

3. Mosser DM, Edwards JP. Exploring the full spectrum of macrophage activation. Nat Rev Immunol. 2008;8(12):958-969.

4. Serbina NV, Jia T, Hohl TM, Pamer EG. Monocytemediated defense against microbial pathogens. Annu Rev Immunol. 2008;26:421-452.

5. Tenen DG. Disruption of differentiation in human cancer: AML shows the way. Nat Rev Cancer. 2003;3(2):89-101.

6 . Gordon S. Alternative activation of macrophages. Nat Rev Immunol. 2003;3(1):23-35.

7. Schroder K, Sweet MJ, Hume DA. Signal integration between IFNgamma and TLR signalling pathways in macrophages. Immunobiology. 2006; 211(6-8):511-524

8. Friedman AD. Transcriptional control of granulocyte and monocyte development. Oncogene. 2007;26(47):6816-6828.

9. Friedman AD. Transcriptional regulation of granulocyte and monocyte development. Oncogene. 2002;21(21):3377-3390.

10. Valledor AF, Borras FE, Cullell-Young M, Celada A. Transcription factors that regulate monocyte/macrophage differentiation. J Leukoc Biol. 1998;63(4):405-417.

11. Tenen DG, Hromas R, Licht JD, Zhang DE. Transcription factors, normal myeloid development, and leukemia. Blood. 1997;90(2):489-519.

12. Yeamans C, Wang D, Paz-Priel I, Torbett BE, Tenen DG, Friedman AD. C/EBPalpha binds and activates the PU.1 distal enhancer to induce monocyte lineage commitment. Blood. 2007;110(9):3136-3142.

13. Scott EW, Simon MC, Anastasi J, Singh H. Requirement of transcription factor PU.1 in the development of multiple hematopoietic lineages. Science. 1994;265(5178):1573-1577.

14. McKercher SR, et al. Targeted disruption of the PU.1 gene results in multiple hematopoietic abnormalities. EMBO J. 1996;15(20):5647-5658.

15. Liu H, Shi B, Huang CC, Eksarko P, Pope RM. Transcriptional diversity during monocyte to macrophage differentiation. Immunol Lett. 2008;117(1):70-80.

16. Martinez FO, Gordon S, Locati M, Mantovani A. Transcriptional profiling of the human monocyteto-macrophage differentiation and polarization: new molecules and patterns of gene expression. J Immunol. 2006;177(10):7303-7311.

17. Lu R, Pitha PM. Monocyte differentiation to macrophage requires interferon regulatory factor 7 . J Biol Chem. 2001;276(48):45491-45496.

18. Chang DH, Angelin-Duclos C, Calame K. BLIMP-1: trigger for differentiation of myeloid lineage. Nat Immunol. 2000;1(2):169-176.

19. Liu M, Lee MH, Cohen M, Bommakanti M, Freedman LP. Transcriptional activation of the Cdk inhibitor $\mathrm{p} 21$ by vitamin D3 leads to the induced differentiation of the myelomonocytic cell line U937. Genes Dev. 1996;10(2):142-153.

20. Gao H, Le Y, Wu X, Silberstein LE, Giese RW, Zhu Z. VentX, a novel lymphoid-enhancing factor/T-cell fac- tor-associated transcription repressor, is a putative tumor suppressor. Cancer Res. 2010;70(1):202-211.

21. Gao H, Wu B, Giese R, Zhu Z. Xom interacts with and stimulates transcriptional activity of LEF1/ TCFs: implications for ventral cell fate determination during vertebrate embryogenesis. Cell Res. 2007;17(4):345-356.

22. Wu X, et al. VentX trans-Activates p53 and p16ink4a to Regulate Cellular Senescence. J Biol Chem. 2011;286(14):12693-12701.

23. Ku M, Howard S, Ni W, Lagna G, Hata A. OAZ regulates bone morphogenetic protein signaling through Smad6 activation. J Biol Chem. 2006;281(8):5277-5287.

24. Rawat VP, et al. The vent-like homeobox gene VENTX promotes human myeloid differentiation and is highly expressed in acute myeloid leukemia. Proc Natl Acad Sci U S A. 2010;107(39):16946-16951.

25. van Grevenynghe J, et al. Polycyclic aromatic hydrocarbons inhibit differentiation of human monocytes into macrophages. J Immunol. 2003; 170(5):2374-2381.

26. Andreesen R, Osterholz J, Bodemann H, Bross KJ, Costabel U, Lohr GW. Expression of transferrin receptors and intracellular ferritin during terminal differentiation of human monocytes. Blut. 1984;49(3):195-202.

27. Scheuerer B, et al. The CXC-chemokine platelet factor 4 promotes monocyte survival and induces monocyte differentiation into macrophages. Blood. 2000;95(4):1158-1166.

28. Rebe C, et al. Caspase-8 prevents sustained activation of NF-kappaB in monocytes undergoing macrophagic differentiation. Blood. 2007;109(4):1442-1450.

29. Gessani S, et al. Enhanced production of LPSinduced cytokines during differentiation of human monocytes to macrophages. Role of LPS receptors. J Immunol. 1993;151(7):3758-3766.

30. Cathelin S, et al. Identification of proteins cleaved downstream of caspase activation in monocytes undergoing macrophage differentiation. J Biol Chem. 2006;281(26):17779-17788.

31. Young DA, Lowe LD, Clark SC. Comparison of the effects of IL-3, granulocyte-macrophage colony-stimulating factor, and macrophage colonystimulating factor in supporting monocyte differentiation in culture. Analysis of macrophage antibody-dependent cellular cytotoxicity. J Immunol. 1990;145(2):607-615.

32. Ragg SJ, Kaga S, Berg KA, Ochi A. The mitogen-activated protein kinase pathway inhibits ceramide-induced terminal differentiation of a human monoblastic leukemia cell line, U937. J Immunol. 1998;161(3):1390-1398.

33. Rovera G, Santoli D, Damsky C. Human promyelocytic leukemia cells in culture differentiate into macrophage-like cells when treated with a phorbol diester. Proc Natl Acad Sci U S A. 1979;76(6):2779-2783.

34. Dai XM, et al. Targeted disruption of the mouse colony-stimulating factor 1 receptor gene results in osteopetrosis, mononuclear phagocyte deficiency, increased primitive progenitor cell frequencies, and reproductive defects. Blood. 2002;99(1):111-120.

35. Laughon A. DNA binding specificity of homeodomains. Biochemistry. 1991;30(48):11357-11367.

36. Bonifer C, Hume DA. The transcriptional regulation of the Colony-Stimulating Factor 1 Receptor (csf1r) gene during hematopoiesis. Front Biosci.
2008:13:549-560

37. Shi C, et al. Integrin engagement regulates monocyte differentiation through the forkhead transcription factor Foxp1. J Clin Invest. 2004;114(3):408-418.

38. Imhof BA, Aurrand-Lions M. Adhesion mechanisms regulating the migration of monocytes. Nat Rev Immunol. 2004;4(6):432-444.

39. Bouhlel MA, et al. PPARgamma activation primes human monocytes into alternative M2 macrophages with anti-inflammatory properties. Cell Metab. 2007;6(2):137-143.

40. Aderem A, Ulevitch RJ. Toll-like receptors in the induction of the innate immune response. Nature. 2000;406(6797):782-787.

41. Hu X, Chen J, Wang L, Ivashkiv LB. Crosstalk among Jak-STAT, Toll-like receptor, and ITAMdependent pathways in macrophage activation. J Leukoc Biol. 2007;82(2):237-243.

42. Schroder K, Hertzog PJ, Ravasi T, Hume DA. Interferon-gamma: an overview of signals, mechanisms and functions. J Leukoc Biol. 2004;75(2):163-189.

43. Feinberg MW, Cao Z, Wara AK, Lebedeva MA, Senbanerjee S, Jain MK. Kruppel-like factor 4 is a mediator of proinflammatory signaling in macrophages. J Biol Chem. 2005;280(46):38247-38258.

44. Das H, et al. Kruppel-like factor 2 (KLF2) regulates proinflammatory activation of monocytes. Proc Natl Acad Sci U S A. 2006;103(17):6653-6658.

45. Cao Z, Sun X, Icli B, Wara AK, Feinberg MW. Role of Kruppel-like factors in leukocyte development, function, and disease. Blood. 2010;116(22):4404-4414.

46. Kawanami D, et al. Kruppel-like factor 2 inhibits hypoxia-inducible factor 1 alpha expression and function in the endothelium. J Biol Chem. 2009;284(31):20522-20530.

47. Lentsch AB, Kato A, Davis B, Wang W, Chao C, Edwards MJ. STAT4 and STAT6 regulate systemic inflammation and protect against lethal endotoxemia. J Clin Invest. 2001;108(10):1475-1482.

48. Murdoch C, Muthana M, Lewis CE. Hypoxia regulates macrophage functions in inflammation. J Immunol. 2005;175(10):6257-6263.

49. Asanuma $Y$, et al. Increased concentration of proatherogenic inflammatory cytokines in systemic lupus erythematosus: relationship to cardiovascular risk factors. J Rheumatol. 2006;33(3):539-545.

50. Sabry A, et al. Proinflammatory cytokines (TNFalpha and IL-6) in Egyptian patients with SLE: its correlation with disease activity. Cytokine. 2006;35(3-4):148-153.

51. Aringer M, Smolen JS. Tumour necrosis factor and other proinflammatory cytokines in systemic lupus erythematosus: a rationale for therapeutic intervention. Lupus. 2004;13(5):344-347.

52. Davas EM, Tsirogianni A, Kappou I, Karamitsos D, Economidou I, Dantis PC. Serum IL-6, TNFalpha, p55 srTNFalpha, p75srTNFalpha, srIL-2alpha levels and disease activity in systemic lupus erythematosus. Clin Rheumatol. 1999;18(1):17-22.

53. Gordon S, Taylor PR. Monocyte and macrophage heterogeneity. Nat Rev Immunol. 2005;5(12):953-964.

54. Feinberg MW, et al. The Kruppel-like factor KLF4 is a critical regulator of monocyte differentiation. Embo J. 2007;26(18):4138-4148.

55. Garcia A, et al. Differential effect on U937 cell differentiation by targeting transcriptional factors implicated in tissue- or stage-specific induced integrin expression. Exp Hematol. 1999;27(2):353-364. 
56. Bourette RP, Rohrschneider LR. Early events in M-CSF receptor signaling. Growth Factors. 2000; 17(3):155-166.

57. Pixley FJ, Stanley ER. CSF-1 regulation of the wandering macrophage: complexity in action. Trends Cell Biol. 2004;14(11):628-638.

58. Chomarat P, Dantin C, Bennett L, Banchereau J, Palucka AK. TNF skews monocyte differentiation from macrophages to dendritic cells. J Immunol. 2003;171(5):2262-2269.

59. Chomarat P, Banchereau J, Davoust J, Palucka AK.
IL- 6 switches the differentiation of monocytes from dendritic cells to macrophages. Nat Immunol. 2000;1(6):510-514.

60. Kramer JL, Baltathakis I, Alcantara OS, Boldt DH. Differentiation of functional dendritic cells and macrophages from human peripheral blood monocyte precursors is dependent on expression of p21 (WAF1/CIP1) and requires iron. BrJ Haematol. 2002;117(3):727-734.

61. Stuart LM, Ezekowitz RA. Phagocytosis: elegant complexity. Immunity. 2005;22(5):539-550.
62. Pringe A, et al. Macrophage activation syndrome in juvenile systemic lupus erythematosus: an under-recognized complication? Lupus. 2007; 16(8):587-592.

63. Gualtierotti R, Biggioggero M, Penatti AE, Meroni PL. Updating on the pathogenesis of systemic lupus erythematosus. Autoimmun Rev. 2010;10(1):3-7.

64. Wu X, Shell SM, Zou Y. Interaction and colocalization of Rad9/Rad1/Hus1 checkpoint complex with replication protein A in human cells. Oncogene. 2005;24(29):4728-4735. 